\title{
Invariance of quantum rings under ordinary flops II: A quantum Leray-Hirsch theorem
}

\author{
Yuan-Pin Lee, Hui-Wen Lin and Chin-Lung Wang
}

\begin{abstract}
This is the second of a sequence of papers proving the quantum invariance for ordinary flops over an arbitrary smooth base. In this paper, we complete the proof of the invariance of the big quantum rings under ordinary flops of split type. To achieve that, several new ingredients are introduced. One is a quantum Leray-Hirsch theorem for the local model (a certain toric bundle) which extends the quantum $\mathscr{D}$-module of the Dubrovin connection on the base by a Picard-Fuchs system of the toric fibers. Non-split flops as well as further applications of the quantum Leray-Hirsch theorem will be discussed in subsequent papers. In particular, a quantum splitting principle is developed in part III (Lee, Lin, Qu and Wang, "Invariance of quantum rings under ordinary flops III", Cambridge Journal of Mathematics, 2016), which reduces the general ordinary flops to the split case solved here.
\end{abstract}

\section{Introduction}

\subsection{Overview}

This paper continues our study of the quantum invariance of genus zero Gromov-Witten theory, up to analytic continuation along the Kähler moduli spaces, under ordinary flops over a nontrivial base. The quantum invariance via analytic continuation plays an important role in the study of various Calabi-Yau compactifications in string theory. It is also a potential tool in comparing various birational minimal models in higher-dimensional algebraic geometry. We refer the readers to [LLW10] and Part I of this series [LLW16] for a general introduction.

In Part I, we determine the defect of the cup product under the canonical correspondence [LLW16, Section 2] and show that it is corrected by the small quantum product attached to the extremal ray [LLW16, Section 3]. We then perform various reductions to local models [LLW16, Sections 4 and 5]. The most important consequence of this reduction is that we may assume that our ordinary flops are between two toric fibrations over the same smooth base.

In this paper, we study the local models via various techniques and complete the proof of the quantum invariance of Gromov-Witten theory in genus zero under ordinary flops of split type.

Received 30 July 2014, accepted in final form 1 February 2016.

2010 Mathematics Subject Classification 14N35, $14 \mathrm{E} 30$.

Keywords: quantum Leray-Hirsch, split ordinary flops, Dubrovin connections, Picard-Fuchs ideal, lifting of QDE, Birkhoff factorization, generalized mirror transform.

This journal is (C) Foundation Compositio Mathematica 2016. This article is distributed with Open Access under the terms of the Creative Commons Attribution Non-Commercial License, which permits non-commercial reuse, distribution, and reproduction in any medium, provided that the original work is properly cited. For commercial re-use, please contact the Foundation Compositio Mathematica.

Y.-P. Lee is partially supported by the NSF; H.-W. Lin is partially supported by the MOST; C.-L. Wang is partially supported by the MOST and the MOE. We are particularly grateful to Taida Institute of Mathematical Sciences (TIMS) for its steady support which makes this long-term collaborative project possible. 
This is, as far as we know, the first result on the quantum invariance under the $K$-equivalence (crepant transformation) [Wan04, Wan03] where the local structure of the exceptional loci cannot be deformed to any explicit (for example, toric) geometry and the analytic continuation is nontrivial. This is also the first result for which the analytic continuation is established with nontrivial Birkhoff factorization.

Several new ingredients are introduced in the course of the proof. One main technical ingredient is the quantum Leray-Hirsch theorem for the local model, which is related to the canonical lift of the quantum $\mathscr{D}$-module from the base to the total space of a (toric) bundle. The techniques developed in this paper are applicable to more general cases and will be discussed in subsequent papers.

Conventions. This paper is strongly correlated with [LLW16], which will be referred to as Part I throughout the paper. All conventions and the notation introduced there carry over to this paper.

\subsection{Outline of the contents}

1.2.1 On the splitting assumption. We recall the local geometry of an ordinary $P^{r}$ flop $f: X \rightarrow X^{\prime}$ (Part I [LLW16, Section 2.1]). The local geometry of the $f$-exceptional loci $Z \subset X$ and $Z^{\prime} \subset X^{\prime}$ is encoded in a triple $\left(S, F, F^{\prime}\right)$, where $S$ is a smooth variety, and $F$ and $F^{\prime}$ are two rank $r+1$ vector bundles over $S$. In Part I [LLW16], we reduce the proof of the invariance of the big quantum ring of any ordinary flop to that of its local model. Therefore, we may assume

$$
\begin{aligned}
X & =\tilde{E}=P_{Z}\left(\mathscr{O}(-1) \otimes F^{\prime} \oplus \mathscr{O}\right), \\
X^{\prime} & =\tilde{E}^{\prime}=P_{Z^{\prime}}(\mathscr{O}(-1) \otimes F \oplus \mathscr{O}),
\end{aligned}
$$

where $Z \cong P_{S}(F)$ and $Z^{\prime} \cong P_{S}\left(F^{\prime}\right)$ are projective bundles. In particular, $X$ and $X^{\prime}$ are toric bundles over the smooth base $S$. Moreover, proving the invariance of the local model is equivalent to proving the type I quasi-linearity property, namely the invariance for 1-pointed descendent fiber series of the form

$$
\left\langle\bar{t}_{1}, \ldots, \bar{t}_{n-1}, \tau_{k} a \xi\right\rangle
$$

where $\bar{t}_{i} \in H(S)$ and $\xi$ is the common infinity divisor of $X$ and $X^{\prime}$.

To proceed, recall that the descendent Gromov-Witten (GW) invariants are encoded by their generating function, that is, the so-called (big) $J$ function: for $\tau \in H(X)$,

$$
J^{X}\left(\tau, z^{-1}\right):=1+\frac{\tau}{z}+\sum_{\beta, n, \mu} \frac{q^{\beta}}{n !} T_{\mu}\left\langle\frac{T^{\mu}}{z(z-\psi)}, \tau, \ldots, \tau\right\rangle_{0, n+1, \beta}^{X} .
$$

The determination of $J$ usually relies on the existence of $\mathbb{C}^{\times}$-action. Certain localization data $I_{\beta}$ coming from the stable map moduli spaces are of hypergeometric type. For "good" cases, say $c_{1}(X)$ is semipositive and $H(X)$ is generated by $H^{2}$, the generating function $I(t)=\sum I_{\beta} q^{\beta}$ determines $J(\tau)$ on the small parameter space $H^{0} \oplus H^{2}$ through the "classical" mirror transform $\tau=\tau(t)$. For a simple flop, $X=X_{\text {loc }}$ is indeed semi-Fano toric and the classical mirror theorem (of Lian-Liu-Yau and Givental) is sufficient [LLW10]. (It turns out that $\tau=t$ and $I=J$ on $H^{0} \oplus H^{2}$.)

For a general base $S$ with given quantum cohomology ring $\mathrm{QH}(S)$, the determination of $\mathrm{QH}(P)$ for a projective bundle $P \rightarrow S$ is far more involved. To allow fiberwise localization to determine the structure of the GW invariants of $X_{\text {loc }}$, the bundles $F$ and $F^{\prime}$ are then assumed to be split bundles. 


\section{INVARIANCE OF QUANTUM RINGS II}

In this paper (Part II), we only consider ordinary flops of split type, namely $F \cong \bigoplus_{i=0}^{r} L_{i}$ and $F^{\prime} \cong \bigoplus_{i=0}^{r} L_{i}^{\prime}$ for some line bundles $L_{i}$ and $L_{i}^{\prime}$ on $S$.

1.2.2 Birkhoff factorization and generalized mirror transformation. The splitting assumption allows one to apply $\mathbb{C}^{\times}$-localization along the fibers of the toric bundle $X \rightarrow S$. Using this and other sophisticated technical tools, Brown (and Givental) [Bro14] proved that the hypergeometric modification

$$
I^{X}\left(D, \bar{t}, z, z^{-1}\right):=\sum_{\beta} q^{\beta} e^{D / z+(D . \beta)} I_{\beta}^{X / S}\left(z, z^{-1}\right) \bar{\psi}^{*} J_{\beta_{S}}^{S}\left(\bar{t}, z^{-1}\right)
$$

lies in Givental's Lagrangian cone generated by $J^{X}\left(\tau, z^{-1}\right)$. Here $D=t^{1} h+t^{2} \xi, \bar{t} \in H(S)$ and $\beta_{S}=\bar{\psi}_{*} \beta$, and the explicit form of $I_{\beta}^{X / S}$ is given in Section 3.2.

Based on Brown's theorem, we prove the following result. (See Section 2 for the notation concerning higher derivatives $\partial^{z \mathbf{e}}$.)

Theorem 1.1 (BF/GMT). There is a unique matrix factorization

$$
\left(\partial^{z \mathbf{e}} I\left(z, z^{-1}\right)\right)=\left(z \nabla J\left(z^{-1}\right)\right) B(z)
$$

called the Birkhoff factorization $(B F)$ of $I$, valid along $\tau=\tau(D, \bar{t}, q)$.

The BF can be stated in another way. There is a recursively defined polynomial differential operator $P(z, q ; \partial)=1+O(z)$ in $t^{1}, t^{2}$ and $\bar{t}$ such that

$$
J\left(z^{-1}\right)=P(z, q ; \partial) I\left(z, z^{-1}\right) .
$$

In other words, $P$ removes the $z$-polynomial part of $I$ in the $\mathrm{NE}(X)$-adic topology. In this form, the generalized mirror transform (GMT)

$$
\tau(D, \bar{t}, q)=D+\bar{t}+\sum_{\beta \neq 0} q^{\beta} \tau_{\beta}(D, \bar{t})
$$

is the coefficient of $z^{-1}$ in $J=P I$.

1.2.3 Hypergeometric modification and $\mathscr{D}$-modules. In principle, knowing the BF, the GMT and the GW invariants on $S$ allows us to calculate all $g=0$ invariants on $X$ and $X^{\prime}$ by reconstruction. These data are in turn encoded in the $I$-functions. One might be tempted to prove the $\mathscr{F}$-invariance by comparing $I^{X}$ and $I^{X^{\prime}}$. While they look rather symmetric, the defect of the cup product implies $\mathscr{F} I^{X} \neq I^{X^{\prime}}$ and the comparison via tracking the defects of the ring isomorphism becomes hopelessly complicated. This can be overcome by studying a more "intrinsic" object: the cyclic $\mathscr{D}$-module $\mathscr{M}_{J}=\mathscr{D} J$, where $\mathscr{D}$ denotes the ring of differential operators on $H$ with suitable coefficients.

It is well known by the topological recursion relations (TRR) that $\left(z \partial_{\mu} J\right)$ forms a fundamental solution matrix of the Dubrovin connection: Namely, we have the quantum differential equations (QDE)

$$
z \partial_{\mu} z \partial_{\nu} J=\sum_{\kappa} \tilde{C}_{\mu \nu}^{\kappa}(t) z \partial_{\kappa} J
$$

where the $\tilde{C}_{\mu \nu}^{\kappa}(t)=\sum_{\iota} g^{\kappa \iota} \partial_{\mu \nu \iota}^{3} F_{0}(t)$ are the structural constants of $*_{t}$. This implies that $\mathscr{M}$ is a holonomic $\mathscr{D}$-module of length $N=\operatorname{dim} H$. For $I$ we consider a similar $\mathscr{D}$-module $\mathscr{M}_{I}=\mathscr{D} I$. Theorem1.1 furnishes a change of basis which implies that $\mathscr{M}_{I}$ is also holonomic of length $N$. 
The idea is to go backward: to first find $\mathscr{M}_{I}$ and then transform it to $\mathscr{M}_{J}$. We do not have similar QDE since $I$ does not have enough variables. Instead we construct higher-order PicardFuchs equations $\square_{\ell} I=0$ and $\square_{\gamma} I=0$ in divisor variables, with the nice property that "up to analytic continuation" they generate $\mathscr{F}$-invariant ideals:

$$
\mathscr{F}\left\langle\square_{\ell}^{X}, \square_{\gamma}^{X}\right\rangle \cong\left\langle\square_{\ell^{\prime}}^{X^{\prime}}, \square_{\gamma^{\prime}}^{X^{\prime}}\right\rangle \text {. }
$$

1.2.4 The quantum Leray-Hirsch theorem and the conclusion of the proof. We now want to determine $\mathscr{M}_{I}$. While the derivatives along the fiber directions are determined by the PicardFuchs equations, we need to find the derivatives along the base direction. Write $\bar{t}=\sum \bar{t}^{i} \bar{T}_{i}$. This is achieved by lifting the QDE on $\mathrm{QH}(S)$, namely

$$
z \partial_{i} z \partial_{j} J^{S}=\sum_{k} \bar{C}_{i j}^{k}(\bar{t}) z \partial_{k} J^{S}
$$

to a differential system on $H(X)$. A key concept needed for such a lift is the $I$-minimal lift of a curve class $\beta_{S} \in \mathrm{NE}(S)$ to $\beta_{S}^{I} \in \mathrm{NE}(X)$. Various lifts of curve classes are discussed in Section 3. See in particular Definition 3.7.

Using the Picard-Fuchs equations and the lifted QDE, we show that $\mathscr{F}_{I^{X}} \cong \mathscr{M}_{I^{X^{\prime}}}$.

Theorem 1.2 (Quantum Leray-Hirsch). (1) (I-lift) The quantum differential equation on $\mathrm{QH}(S)$ can be lifted to $H(X)$ as

$$
z \partial_{i} z \partial_{j} I=\sum_{k, \beta_{S}} q^{\beta_{S}^{I}} e^{\left(D \cdot \beta_{S}^{I}\right)} \bar{C}_{i j, \beta_{S}}^{k}(\bar{t}) z \partial_{k} D_{\beta_{S}^{I}}(z) I
$$

where $D_{\beta_{S}^{I}}(z)$ is an operator depending only on $\beta_{S}^{I}$. Any other lift is related to it modulo the Picard-Fuchs system.

(2) Together with the Picard-Fuchs operators $\square_{\ell}$ and $\square_{\gamma}$, the QDE determine a first-order matrix system under the naive quantization $\partial^{z \mathbf{e}}$ (Definition 4.7) of the canonical basis $T_{\mathbf{e}}$ (Notation 4.1) of $H(X)$ :

$$
z \partial_{a}\left(\partial^{z \mathbf{e}} I\right)=\left(\partial^{z \mathbf{e}} I\right) C_{a}(z, q), \quad \text { where } t^{a}=t^{1}, t^{2} \text { or } \bar{t}^{i}
$$

(3) The system has the property that for any fixed $\beta_{S} \in \mathrm{NE}(S)$, the coefficients are formal functions in $\bar{t}$ and polynomial functions in $q^{\gamma} e^{t^{2}}, q^{\ell} e^{t^{1}}$ and $\mathbf{f}\left(q^{\ell} e^{t^{1}}\right)$. Here the basic rational function

$$
\mathbf{f}(q):=q /\left(1-(-1)^{r+1} q\right)
$$

is the "origin of analytic continuation" satisfying $\mathbf{f}(q)+\mathbf{f}\left(q^{-1}\right)=(-1)^{r}$.

(4) The system is $\mathscr{F}$-invariant.

The final step is to go from $\mathscr{M}_{I}$ to $\mathscr{M}_{J}$. From the perspective of $\mathscr{D}$-modules, the BF can be considered as a gauge transformation. The defining property $\left(\partial^{z \mathbf{e}} I\right)=(z \nabla J) B$ of $B$ can be rephrased as

$$
z \partial_{a}(z \nabla J)=(z \nabla J) \tilde{C}_{a}
$$

so that

$$
\tilde{C}_{a}=\left(-z \partial_{a} B+B C_{a}\right) B^{-1}
$$

is independent of $z$.

This formulation has the advantage that all objects in (1.2) are expected to be $\mathscr{F}$-invariant (while $I$ and $J$ are not). It is therefore easier to first establish the $\mathscr{F}$-invariance of the $C_{a}$ and use 


\section{INVARIANCE OF QUANTUM RINGS II}

it to derive the $\mathscr{F}$-invariance of the BF and GMT. As a consequence, this allows us to deduce the type I quasi-linearity (Proposition 2.11), and hence the invariance of the big quantum rings for local models.

Theorem 1.3 (Quantum invariance). For ordinary flops of split type, the big quantum cohomology ring is invariant up to analytic continuation.

By the reduction procedure in Part I [LLW16], this is equivalent to the quasi-linearity property of the local models. This completes the outline.

Remark 1.4. Results in this paper had been announced by the authors, in increasing degree of generality, at various conferences during 2008-2012; see, for example, [Lin10, Wan11, LLW12], where more examples are studied. Examples of the quantum Leray-Hirsch theorem are included in Section 5. The complete proofs of Theorems 1.2 and 1.3 were achieved mid-2011.

It might seem possible to prove Theorem 1.3 directly from comparisons of $J$-functions and Birkhoff factorizations on $X$ and $X^{\prime}$. Indeed, we were able to carry this out for various special cases. A mysterious regularization phenomenon appears during such a direct approach. In the appendix we explain how the regularization of certain rational functions leads to the beginning steps of analytic continuations in our context. However, the combinatorial complexity becomes intractable (to us) in the general case. Some examples can be found in the proceedings articles referred to above.

In Part III [LLQW16], the final part of this series, we will develop a quantum splitting principle to remove the splitting assumption in Theorem 1.3. This then completes our study of the quantum invariance under ordinary flops.

\section{Birkhoff factorization}

In this section, a general framework for calculating the $J$-function for a split toric bundle is discussed. It relies on a given (partial) section $I$ of the Lagrangian cone generated by $J$. The process to go from $I$ to $J$ is introduced in a constructive manner, and Theorem 1.1 will be proved (as the combination of Proposition 2.6 and Theorem 2.10).

\subsection{Lagrangian cone and the $J$-function}

We start with Givental's symplectic space reformulation of Gromov-Witten theory arising from the dilaton, string and topological recursion relation. The main references for this section are [Giv04, CG07], with supplements and clarification from [LP, Lee09]. In the following, the underlying ground ring is the Novikov ring

$$
R=\mathbb{C}[\widehat{\operatorname{NE}(X)}] .
$$

All complicated issues on completion are deferred to [LP].

Let $H:=H(X), \mathcal{H}:=H\left[z, z^{-1} \rrbracket, \mathcal{H}_{+}:=H[z]\right.$ and $\mathcal{H}_{-}:=z^{-1} H \llbracket z^{-1} \rrbracket$. Let $1 \in H$ be the identity. One can identify $\mathcal{H}$ as $T^{*} \mathcal{H}_{+}$, and this gives a canonical symplectic structure and a vector bundle structure on $\mathcal{H}$.

Let

$$
\mathbf{q}(z)=\sum_{\mu} \sum_{k=0}^{\infty} \mathbf{q}_{k}^{\mu} T_{\mu} z^{k} \in \mathcal{H}_{+}
$$


be a general point, where $\left\{T_{\mu}\right\}$ forms a basis of $H$. In the Gromov-Witten context, the natural coordinates on $\mathcal{H}_{+}$are $\mathbf{t}(z)=\mathbf{q}(z)+1 z$ (dilaton shift), with $\mathbf{t}(\psi)=\sum_{\mu, k} t_{k}^{\mu} T_{\mu} \psi^{k}$ serving as the general descendent insertion. Let $F_{0}(\mathbf{t})$ be the generating function of the genus zero descendent Gromov-Witten invariants on $X$. Since $F_{0}$ is a function on $\mathcal{H}_{+}$, the 1 -form $d F_{0}$ gives a section of $\pi: \mathcal{H} \rightarrow \mathcal{H}_{+}$.

Givental's Lagrangian cone $\mathcal{L}$ is defined as the graph of $d F_{0}$, which is considered as a section of $\pi$. By construction it is a Lagrangian subspace. The existence of $\mathbb{C}^{*}$-action on $\mathcal{L}$ is due to the dilaton equation $\sum \mathbf{q}_{k}^{\mu} \partial / \partial \mathbf{q}_{k}^{\mu} F_{0}=2 F_{0}$. Thus $\mathcal{L}$ is a cone with vertex $\mathbf{q}=0$ (cf. [Giv04, Lee09]).

Let $\tau=\sum_{\mu} \tau^{\mu} T_{\mu} \in H$. Define the (big) $J$-function to be

$$
\begin{aligned}
J^{X}\left(\tau, z^{-1}\right) & =1+\frac{\tau}{z}+\sum_{\beta, n, \mu} \frac{q^{\beta}}{n !} T_{\mu}\left\langle\frac{T^{\mu}}{z(z-\psi)}, \tau, \ldots, \tau\right\rangle_{0, n+1, \beta} \\
& =e^{\tau / z}+\sum_{\beta \neq 0, n, \mu} \frac{q^{\beta}}{n !} e^{\tau_{1} / z+\left(\tau_{1} . \beta\right)} T_{\mu}\left\langle\frac{T^{\mu}}{z(z-\psi)}, \tau_{2}, \ldots, \tau_{2}\right\rangle_{0, n+1, \beta},
\end{aligned}
$$

where in the second expression $\tau=\tau_{1}+\tau_{2}$ with $\tau_{1} \in H^{2}$. The equality follows from the divisor equation for descendent invariants. Furthermore, the string equation for $J^{X}$ says that we can take out the fundamental class 1 from the variable $\tau$ to get an overall factor $e^{\tau^{0} / z}$ in front of (2.1).

The $J$-function can be considered as a map from $H$ to $z \mathcal{H}_{-}$. Let $L_{\mathbf{f}}=T_{\mathbf{f}} \mathcal{L}$ be the tangent space of $\mathcal{L}$ at $\mathbf{f} \in \mathcal{L}$. Let $\tau \in H$ be embedded into $\mathcal{H}_{+}$via

$$
H \cong-1 z+H \subset \mathcal{H}_{+} \text {. }
$$

Set $L_{\tau}=L_{\left(\tau, d F_{0}(\tau)\right)}$. Here we list the basic structural results from [Giv04]:

(i) We have $z L_{\tau} \subset L_{\tau}$, and so $L_{\tau} / z L_{\tau} \cong \mathcal{H}_{+} / z \mathcal{H}_{+} \cong H$ has $\operatorname{rank} N:=\operatorname{dim} H$.

(ii) We have $L_{\tau} \cap \mathcal{L}=z L_{\tau}$, considered as subspaces inside $\mathcal{H}$.

(iii) The subspace $L_{\tau}$ of $\mathcal{H}$ is the tangent space at every $\mathbf{f} \in z L_{\tau} \subset \mathcal{L}$. Moreover, $T_{\mathbf{f}}=L_{\tau}$ implies $\mathbf{f} \in z L_{\tau}$. The subspace $z L_{\tau}$ is considered as the ruling of the cone.

(iv) The intersection of $\mathcal{L}$ and the affine space $-1 z+z \mathcal{H}_{-}$is parameterized by its image $-1 z+$ $H \cong H$ via the projection by $\pi$. For $\tau \in H$,

$$
-z J^{X}\left(\tau,-z^{-1}\right)=-1 z+\tau+O(1 / z)
$$

is the function of $\tau$ whose graph is the intersection.

(v) The set of all directional derivatives $z \partial_{\mu} J^{X}=T_{\mu}+O(1 / z)$ spans an $N$-dimensional subspace of $L_{\tau}$, namely $L_{\tau} \cap z \mathcal{H}_{-}$, such that its projection to $L_{\tau} / z L_{\tau}$ is an isomorphism.

Note that we have used a convention for the $J$-function which differs from that of some more recent papers [Giv04, CG07] by a factor $z$.

Lemma 2.1. The matrix $z \nabla J^{X}=\left(z \partial_{\mu} J^{\nu}\right)$ has column vectors $z \partial_{\mu} J^{X}(\tau)$ that generate the tangent space $L_{\tau}$ of the Lagrangian cone $\mathcal{L}$ as an $R\{z\}$-module. Here $a=\sum q^{\beta} a_{\beta}(z) \in R\{z\}$ if $a_{\beta}(z) \in \mathbb{C}[z]$.

Proof. Apply result (v) above to $L_{\tau} / z L_{\tau}$ and multiply by $z^{k}$ to get $z^{k} L_{\tau} / z^{k+1} L_{\tau}$.

We see that the germ of $\mathcal{L}$ is determined by an $N$-dimensional submanifold. In this sense, $z J^{X}$ generates $\mathcal{L}$. Indeed, all discussions are applicable to the Gromov-Witten context only as formal germs around the neighborhood of $\mathbf{q}=-1 z$. 


\section{INVARIANCE OF QUANTUM RINGS II}

\subsection{Generalized mirror transform for toric bundles}

Let $\bar{p}: X \rightarrow S$ be a smooth fiber bundle such that $H(X)$ is generated by $H(S)$ and fiber divisors $D_{i}$ as an algebra, in such a way that there is no linear relation among the $D_{i}$ and $H^{2}(S)$. An example of $X$ is a toric bundle over $S$. Assume that $H(X)$ is a free module over $H(S)$ with finite generators $\left\{D^{e}:=\prod_{i} D_{i}^{e_{i}}\right\}_{e \in \Lambda^{+}}$.

Let $\bar{t}:=\sum_{s} \bar{t}^{s} \bar{T}_{s}$ be a general cohomology class in $H(S)$, which is identified with $\bar{p}^{*} H(S)$. Similarly, denote by $D=\sum t^{i} D_{i}$ the general fiber divisor. Elements in $H(X)$ can be written as linear combinations of $\left\{T_{(s, e)}=\bar{T}_{s} D^{e}\right\}$. Denote the $\bar{T}_{s}$-directional derivative on $H(S)$ by $\partial_{\bar{T}_{s}} \equiv \partial_{\bar{t}^{s}}$, and denote the multiple derivative by

$$
\partial^{(s, e)}:=\partial_{\bar{t}^{s}} \prod_{i} \partial_{t^{i}}^{e_{i}}
$$

Note, however, that most of the time $z$ will appear with derivative. For notational convenience, denote the index $(s, e)$ by e. We then set

$$
\partial^{z \mathbf{e}} \equiv \partial^{z(s, e)}:=z \partial_{\bar{t}^{s}} \prod_{i} z \partial_{t^{i}}^{e_{i}}=z^{|e|+1} \partial^{(s, e)} .
$$

As usual, the $T_{\mathbf{e}}$-directional derivative on $H(X)$ is denoted by $\partial_{\mathbf{e}}=\partial_{T_{\mathbf{e}}}$. Here $T_{\mathbf{e}}$ is a special choice of the basis $T_{\mu}$ (and $\partial_{\mu}$ ) of $H(X)$, defined by

$$
T_{\mathbf{e}} \equiv T_{(s, e)} \equiv \bar{T}_{s} D^{e}, \quad \mathbf{e} \in \Lambda^{+} .
$$

The two operators $\partial^{z \mathbf{e}}$ and $z \partial_{\mathbf{e}}$ are by definition very different; nevertheless, they are closely related in the study of quantum cohomology, as we will see below.

Assume that $\bar{p}: X \rightarrow S$ is a toric bundle of split type, that is, the toric quotient of a split vector bundle over $S$. Let $J^{S}\left(\bar{t}, z^{-1}\right)$ be the $J$-function on $S$. The hypergeometric modification of $J^{S}$ by the $\bar{p}$-fibration takes on the form

$$
I^{X}\left(\bar{t}, D, z, z^{-1}\right):=\sum_{\beta \in \operatorname{NE}(X)} q^{\beta} e^{\frac{D}{z}+(D . \beta)} I_{\beta}^{X / S}\left(z, z^{-1}\right) J_{\beta_{S}}^{S}\left(\bar{t}, z^{-1}\right),
$$

with the relative factor $I_{\beta}^{X / S}$, whose explicit form for $X=\tilde{E} \rightarrow S$ will be given in Section 3.2.

The major difficulty which makes $I^{X}$ deviate from $J^{X}$ lies in the fact that in general, positive $z$-powers may occur in $I^{X}$. Nevertheless, for each $\beta \in \mathrm{NE}(X)$ the power of $z$ in $I_{\beta}^{X / S}\left(z, z^{-1}\right)$ is bounded from above by a constant depending only on $\beta$. Thus we may study $I^{X}$ in the space $\mathcal{H}:=H\left[z, z^{-1} \rrbracket\right.$ over $R$.

Notice that the $I$-function is defined only on the subspace

$$
\hat{t}:=\bar{t}+D \in H(S) \oplus \bigoplus_{i} \mathbb{C} D_{i} \subset H(X) .
$$

We will use the following theorem by Brown (and Givental).

TheOREM 2.2 ([Bro14, Theorem 1]). The vector $(-z) I^{X}(\hat{t},-z)$ lies in the Lagrangian cone $\mathcal{L}$ of $X$.

Definition 2.3 (GMT). For each $\hat{t}$, the vector $z I(\hat{t})$ lies in the subspace $L_{\tau}$ of $\mathcal{L}$. The correspondence

$$
\hat{t} \mapsto \tau(\hat{t}) \in H(X) \otimes R
$$

is called the generalized mirror transformation (cf. [CG07, Giv04]). 
Remark 2.4. In general $\tau(\hat{t})$ may be outside the submodule of the Novikov ring $R$ generated by $H(S) \oplus \bigoplus_{i} \mathbb{C} D_{i}$. This is in contrast to the (classical) mirror transformation where $\tau$ is a transformation within $\left(H^{0}(X) \oplus H^{2}(X)\right)_{R}$ (small parameter space).

To use Theorem 2.2, we start by outlining the idea behind the following discussions. By the properties of $\mathcal{L}$, Theorem 2.2 implies that $I$ can be obtained from $J$ by applying a certain differential operator in the $z \partial_{\mathbf{e}}$ to it, with series in $z$ as coefficients. However, what we need is the reverse direction, namely to obtain $J$ from $I$, which amounts to removing the positive $z$-powers from $I$. Note that the $I$-function has variables only in the subspace $H(S) \oplus \bigoplus_{i} \mathbb{C} D_{i}$. Thus a priori the reverse direction does not seem to be possible.

The key idea below is to replace derivatives in the missing directions by higher-order differentiations in the fiber divisor variables $t^{i}$, a process similar to transforming a first-order ordinary differential equation system to a higher-order scalar equation. This is possible since $H(X)$ is generated by the $D_{i}$ as an algebra over $H(S)$.

Lemma 2.5. We have $z \partial_{1} J^{X}=J^{X}$ and $z \partial_{1} I^{X}=I^{X}$.

Proof. The first equality is the string equation. For the second one, by definition

$$
I^{X}=\sum_{\beta} q^{\beta} e^{D / z+(D \cdot \beta)} I_{\beta}^{X / S} J_{\beta_{S}}^{S}(\bar{t}),
$$

where $I_{\beta}^{X / S}$ depends only on $z$. The differentiation with respect to $t^{0}$ (dual coordinate of 1 ) only applies to $J_{\beta_{S}}^{S}(\bar{t})$. Hence the string equation on $J_{\beta_{S}}^{S}(\bar{t})$ concludes the proof.

To avoid cluttered notation, we use $I$ and $J$ to denote the $I$-function and $J$-function, respectively, when the target space is clear.

Proposition 2.6. (1) The GMT $\tau=\tau(\hat{t})$ satisfies $\tau(\hat{t}, q=0)=\hat{t}$.

(2) Under the basis $\left\{T_{\mathbf{e}}\right\}_{\mathbf{e} \in \Lambda^{+}}$, there exists an invertible formal series $B(\tau, z)$ with $N \times N$ matrices as coefficients, which is free from cohomology classes, such that

$$
\left(\partial^{z \mathbf{e}} I\left(\hat{t}, z, z^{-1}\right)\right)=\left(z \nabla J\left(\tau, z^{-1}\right)\right) B(\tau, z),
$$

where $\left(\partial^{z \mathbf{e}} I\right)$ is the $N \times N$ matrix with $\partial^{z \mathbf{e}} I$ as the column vectors.

Proof. By Theorem 2.2, we have $z I \in \mathcal{L}$, hence $z \partial I \in T \mathcal{L}=L$. Then $z(z \partial) I \in z L \subset \mathcal{L}$ and so $z \partial(z \partial) I$ lies again in $L$. Inductively, $\partial^{z \mathbf{e}} I$ lies in $L$. The factorization $\left(\partial^{z \mathbf{e}} I\right)=(z \nabla J) B(z)$ then follows from Lemma 2.1. Also, Lemma 2.5 says that the $I$ - and $J$-functions appear as the first column vectors of $\left(\partial^{z \mathbf{e}} I\right)$ and $(z \nabla J)$, respectively. By the $R\{z\}$-module structure, it is clear that $B$ does not involve any cohomology classes.

By the definitions of $J, I$ and $\partial^{z e}$ (cf. (2.1), (2.3), (2.2)), it is clear that

$$
\partial^{z \mathbf{e}} e^{\hat{t} / z}=T_{\mathbf{e}} e^{\hat{t} / z}, \quad z \partial_{\mathbf{e}} e^{t / z}=T_{\mathbf{e}} e^{t / z}
$$

$(t \in H(X))$. Hence modulo Novikov variables, $\partial^{z \mathbf{e}} I(\hat{t}) \equiv T_{\mathbf{e}} e^{\hat{t} / z}$ and $z \partial_{\mathbf{e}} J(\tau) \equiv T_{\mathbf{e}} e^{\tau / z}$.

To prove statement (1), note that modulo all $q^{\beta}$ we have

$$
e^{\hat{t} / z} \equiv \sum_{\mathbf{e} \in \Lambda^{+}} B_{\mathbf{e}, 1}(z) T_{\mathbf{e}} e^{\tau(\hat{t}) / z} .
$$

Thus

$$
e^{(\hat{t}-\tau(\hat{t})) / z} \equiv \sum_{\mathbf{e}} B_{\mathbf{e}, 1}(z) T_{\mathbf{e}}
$$




\section{INVARIANCE OF QUANTUM RINGS II}

which forces $\tau(\hat{t}) \equiv \hat{t}$ (and $\left.B_{\mathbf{e}, 1}(z) \equiv \delta_{T_{\mathbf{e}}, 1}\right)$.

To prove statement (2), notice that by statement (1) and (2.6), we have $B(\tau, z) \equiv I_{N \times N}$ modulo Novikov variables, so in particular $B$ is invertible. Notice that in deriving (2.5) we do not need to worry about the sign on " $-z$ " since it appears in both $I$ and $J$.

Definition $2.7(\mathrm{BF})$. The left-hand side of (2.5) involves $z$ and $z^{-1}$, while the right-hand side is the product of a function of $z$ and a function of $z^{-1}$. Such a matrix-factorization process is termed a Birkhoff factorization.

Besides its existence and uniqueness, for actual computations it will be important to know how to calculate $\tau(\hat{t})$ directly or inductively.

Proposition 2.8. There are scalar-valued formal series $C_{\mathbf{e}}(\hat{t}, z)$ such that

$$
J\left(\tau, z^{-1}\right)=\sum_{\mathbf{e} \in \Lambda^{+}} C_{\mathbf{e}}(\hat{t}, z) \partial^{z \mathbf{e}} I\left(\hat{t}, z, z^{-1}\right),
$$

where $C_{\mathbf{e}} \equiv \delta_{T_{\mathbf{e}}, 1}$ modulo Novikov variables.

In particular, $\tau(\hat{t})=\hat{t}+\cdots$ is determined by the coefficient of $1 / z$ on the right-hand side.

Proof. Proposition 2.6 implies

$$
z \nabla J=\left(\partial^{z \mathbf{e}} I\right) B^{-1} .
$$

Take the first column vector on the left-hand side, which is $z \nabla_{1} J=J$ by Lemma 2.5. One gets expression (2.7) by defining $C_{\mathbf{e}}$ to be the corresponding eth entry of the first column vector of $B^{-1}$. Modulo the $q^{\beta}$, we have $B^{-1} \equiv I_{N \times N}$, hence $C_{\mathbf{e}} \equiv \delta_{T_{\mathbf{e}}, 1}$.

Definition 2.9. A differential operator $P$ is of degree $\Lambda^{+}$if $P=\sum_{\mathbf{e} \in \Lambda^{+}} C_{\mathbf{e}} \partial^{z \mathbf{e}}$ for some $C_{\mathbf{e}}$. Namely, its components are multi-derivatives indexed by $\Lambda^{+}$.

TheOrem 2.10 (BF/GMT). There is a unique, recursively determined, scalar-valued degree $\Lambda^{+}$ differential operator

$$
P(z)=1+\sum_{\beta \in \operatorname{NE}(X) \backslash\{0\}} q^{\beta} P_{\beta}\left(t^{i}, \bar{t}^{s}, z ; z \partial_{t^{i}}, z \partial_{\bar{t}^{s}}\right),
$$

with each $P_{\beta}$ polynomial in $z$, such that $P(z) I\left(\hat{t}, z, z^{-1}\right)=1+O(1 / z)$.

Moreover,

$$
J\left(\tau(\hat{t}), z^{-1}\right)=P(z) I\left(\hat{t}, z, z^{-1}\right),
$$

with $\tau(\hat{t})$ determined by the coefficient of $1 / z$ on the right-hand side.

Proof. The operator $P(z)$ is constructed by induction on $\beta \in \mathrm{NE}(X)$. We set $P_{\beta}=1$ for $\beta=0$. Suppose that $P_{\beta^{\prime}}$ has been constructed for all $\beta^{\prime}<\beta$ in $\mathrm{NE}(X)$. We set $P_{<\beta}(z)=\sum_{\beta^{\prime}<\beta} q^{\beta^{\prime}} P_{\beta^{\prime}}$. Let

$$
A_{1}=z^{k_{1}} q^{\beta} \sum_{\mathbf{e} \in \Lambda^{+}} f^{\mathbf{e}}\left(t^{i}, \bar{t}^{s}\right) T_{\mathbf{e}}
$$

be the top $z$-power term in $P_{<\beta}(z) I$. If $k_{1}<0$, then we are done. Otherwise we will remove it by introducing "certain $P_{\beta}$ ". Consider the "naive quantization"

$$
\hat{A}_{1}:=z^{k_{1}} q^{\beta} \sum_{\mathbf{e} \in \Lambda^{+}} f^{\mathbf{e}}\left(t^{i}, \bar{t}^{s}\right) \partial^{z \mathbf{e}} .
$$


In the expression

$$
\left(P_{<\beta}(z)-\hat{A}_{1}\right) I=P_{<\beta}(z) I-\hat{A}_{1} I
$$

the target term $A_{1}$ has been removed since

$$
\hat{A}_{1} I(q=0)=\hat{A}_{1} e^{\hat{t} / z}=A_{1} e^{\hat{t} / z}=A_{1}+A_{1} O(1 / z) .
$$

All the newly created terms either have smaller top $z$-power or have curve degree $q^{\beta^{\prime \prime}}$ with $\beta^{\prime \prime}>\beta$ in $\mathrm{NE}(X)$. Thus we may keep on removing the new top $z$-power term $A_{2}$, which has $k_{2}<k_{1}$. Since the process will stop in no more than $k_{1}$ steps, we simply define $P_{\beta}$ by

$$
q^{\beta} P_{\beta}=-\sum_{1 \leqslant j \leqslant k_{1}} \hat{A}_{j}
$$

By induction we get $P(z)=\sum_{\beta \in \mathrm{NE}(X)} q^{\beta} P_{\beta}$, which is clearly of degree $\Lambda^{+}$.

Now we prove the uniqueness of $P(z)$. Suppose that $P_{1}(z)$ and $P_{2}(z)$ are two such operators. The difference $\delta(z)=P_{1}(z)-P_{2}(z)$ satisfies

$$
\delta(z) I=: \sum_{\beta} q^{\beta} \delta_{\beta} I=O(1 / z) .
$$

Clearly $\delta_{0}=0$. If $\delta_{\beta} \neq 0$ for some $\beta$, then $\beta$ can be chosen such that $\delta_{\beta^{\prime}}=0$ for all $\beta^{\prime}<\beta$. Let the highest non-zero $z$-power term of $\delta_{\beta}$ be $z^{k} \sum_{\mathbf{e}} \delta_{\beta, k, \mathbf{e}} \partial^{z \mathbf{e}}$. Then

$$
q^{\beta} z^{k} \sum_{\mathbf{e}} \delta_{\beta, k, \mathbf{e}} \partial^{z \mathbf{e}}\left(e^{\hat{t} / z}+\sum_{\beta_{1} \neq 0} q^{\beta_{1}} I_{\beta_{1}}\right)+R I=O(1 / z) .
$$

Here $R$ denotes the remaining terms in $\delta$. Note that terms in $R I$ either do not contribute to $q^{\beta}$ or have $z$-power less than $k$. Thus the only $q^{\beta}$-term is

$$
q^{\beta} z^{k} \sum_{\mathbf{e}} \delta_{\beta, k, \mathbf{e}} T_{\mathbf{e}} e^{\hat{t} / z}
$$

This is impossible since $k \geqslant 0$ and $\left\{T_{\mathbf{e}}\right\}$ is a basis. Thus $\delta=0$.

Finally, by Lemma 2.1, both $B$ and $B^{-1}$ have entries in $R\{z\}$. Thus Proposition 2.8 provides an operator which satisfies the required properties. By the uniqueness it must coincide with the effectively constructed $P(z)$.

\subsection{Reduction to special BF/GMT}

Proposition 2.11. Let $f: X \rightarrow X^{\prime}$ be the projective local model of an ordinary flop with graph correspondence $\mathscr{F}$. Suppose that there are formal lifts $\tau, \tau^{\prime}$ of $\hat{t}$ in $H(X) \otimes R$ and $H\left(X^{\prime}\right) \otimes R$, respectively, with $\tau(\hat{t}), \tau^{\prime}(\hat{t}) \equiv \hat{t}$ modulo Novikov variables in $\mathrm{NE}(S)$, and with $\mathscr{F} \tau(\hat{t}) \cong \tau^{\prime}(\hat{t})$. Then

$$
\mathscr{F} J(\tau(\hat{t})) \cdot \xi \cong J^{\prime}\left(\tau^{\prime}(\hat{t})\right) \cdot \xi^{\prime} \Longrightarrow \mathscr{F} J(\hat{t}) \cdot \xi \cong J^{\prime}(\hat{t}) \cdot \xi^{\prime},
$$

and consequently $\mathrm{QH}(X)$ and $\mathrm{QH}\left(X^{\prime}\right)$ are analytic continuations of each other under $\mathscr{F}$.

Proof. For an induction on the weight $w:=\left(\beta_{S}, d_{2}\right) \in W$, suppose that for all $w^{\prime}<w$ we have the invariance of any $n$-point function (except that if $\beta_{S}^{\prime}=0$, then $n \geqslant 3$ ). Here we would like to recall that $W:=(\mathrm{NE}(\tilde{E}) / \sim) \subset \mathrm{NE}(S) \oplus \mathbb{Z}$ is the quotient Mori cone.

By the definition of $J$ in $(2.1)$, for any $a \in H(X)$ we may pick up the fiber series over $w$ from the $\xi a z^{-(k+2)}$-component of the assumed $\mathscr{F}$-invariance:

$$
\mathscr{F}\left\langle\tau^{n}, \psi^{k} \xi a\right\rangle^{X} \cong\left\langle\tau^{\prime n}, \psi^{k} \xi^{\prime} \mathscr{F} a\right\rangle^{X^{\prime}}
$$




\section{INVARIANCE OF QUANTUM RINGS II}

Write $\tau(\hat{t})=\sum_{\bar{w} \in W} \tau_{\bar{w}}(\hat{t}) q^{\bar{w}}$. The fiber series is decomposed into a sum of subseries in $q^{\ell}$ of the form

$$
\left\langle\tau_{\bar{w}_{1}}(\hat{t}), \ldots, \tau_{\bar{w}_{n}}(\hat{t}), \psi^{k} \xi a\right\rangle_{w^{\prime \prime}}^{X} q^{\sum_{j=1}^{n} \bar{w}_{j}+w^{\prime \prime}} .
$$

Since $\sum \bar{w}_{j}+w^{\prime \prime}=w$, any $\bar{w}_{j}$-term with $\bar{w}_{j} \neq 0$ leads to $w^{\prime \prime}<w$, whose fiber series is of the form $\sum_{i} g_{i}\left(q^{\ell}, \hat{t}\right) h_{i}\left(q^{\ell}\right)$ with $g_{i}$ from $\prod \tau_{\bar{w}_{j}}(\hat{t})$ and $h_{i}$ a fiber series over $w^{\prime \prime}$. The $g_{i}$ are $\mathscr{F}$ invariant by assumption and the $h_{i}$ are $\mathscr{F}$-invariant by induction, thus the sum of products is also $\mathscr{F}$-invariant.

From (2.10) and $\tau_{0}(\hat{t})=\hat{t}$, the remaining fiber series with $\bar{w}_{j}=0$ for all $j$ satisfies

$$
\mathscr{F}\left\langle\hat{t}^{n}, \psi^{k} \xi a\right\rangle_{w}^{X} \cong\left\langle\hat{t}^{n}, \psi^{k} \xi \mathscr{F} a\right\rangle_{w^{\prime}}^{X^{\prime}},
$$

which holds for any $n, k$ and $a$.

Now by Theorem 5.2 (divisorial reconstruction and WDVV reduction) of Part I [LLW16], this implies the $\mathscr{F}$-invariance of all fiber series over $w$.

Later we will see that for the GMT $\tau(\hat{t})$ and $\tau^{\prime}(\hat{t})$, the lifting condition $\tau(\hat{t}) \equiv \hat{t}$ modulo $\mathrm{NE}(S) \backslash\{0\}$ (instead of modulo $\mathrm{NE}(X) \backslash\{0\})$ and the identity $\mathscr{F} J(\tau(\hat{t})) \cdot \xi \cong J^{\prime}\left(\tau^{\prime}(\hat{t})\right) \cdot \xi^{\prime}$ hold for split ordinary flops.

\section{Hypergeometric modification}

From now on we work with a split local $P^{r}$ flop $f: X \rightarrow X^{\prime}$ with bundle data $\left(S, F, F^{\prime}\right)$, where

$$
F=\bigoplus_{i=0}^{r} L_{i} \quad \text { and } \quad F^{\prime}=\bigoplus_{i=0}^{r} L_{i}^{\prime} .
$$

We study the explicit formulae of the hypergeometric modifications $I^{X}$ and $I^{X^{\prime}}$ associated with the double projective bundles $X \rightarrow S$ and $X^{\prime} \rightarrow S$, especially the symmetry property between them.

In order to get a better sense of the factor $I^{X / S}$, it is necessary to have a precise description of the Mori cone first. We then describe the Picard-Fuchs equations associated with the $I$-function.

\subsection{Minimal lift of curve classes and $\mathscr{F}$-effective cone}

Let $C$ be an irreducible projective curve with $\psi: V=\bigoplus_{i=0}^{r} \mathscr{O}\left(\mu_{i}\right) \rightarrow C$ a split bundle. Let $\mu=\max \mu_{i}$, and denote by $\bar{\psi}: P(V) \rightarrow C$ the associated projective bundle. Let $h=c_{1}\left(\mathscr{O}_{P(V)}(1)\right)$, let

$$
b=\bar{\psi}^{*}[C] \cdot H_{r}=H_{r}=h^{r}+c_{1}(V) h^{r-1}
$$

be the canonical lift of the base curve, and $\ell$ be the fiber curve class.

Lemma 3.1. The Mori cone $\mathrm{NE}(P(V))$ is generated by $\ell$ and $b-\mu \ell$.

Proof. Consider $V^{\prime}=\mathscr{O}(-\mu) \otimes V=\mathscr{O} \oplus N$. Then $N$ is a semi-negative bundle and $\mathrm{NE}(P(V)) \cong$ $\operatorname{NE}\left(P\left(V^{\prime}\right)\right)$ is generated by $\ell$ and the zero section $b^{\prime}$ of $N \rightarrow P^{1}$. In this case $b^{\prime}$ is also the canonical lift $b^{\prime}=h^{\prime r}+c_{1}\left(V^{\prime}\right) h^{\prime r-1}$. From the Euler sequence we know that $h^{\prime}=h+\mu p$. Hence

$$
\begin{aligned}
b^{\prime} & =(h+\mu p)^{r}+\sum_{i=1}^{r}\left(\mu_{i}-\mu\right) p(h+\mu p)^{r+1}=h^{r}+r \mu p h^{r-1}+\sum_{i=1}^{r}\left(\mu_{i}-\mu\right) p h^{r-1} \\
& =h^{r}+c_{1}(V) h^{r-1}-\mu p h^{r-1}=b-\mu \ell .
\end{aligned}
$$


Let $\psi: V=\bigoplus_{i=0}^{r} L_{i} \rightarrow S$ be a split bundle with $\bar{\psi}: P=P(V) \rightarrow S$. Since $\bar{\psi}_{*}: \mathrm{NE}(P) \rightarrow$ $\mathrm{NE}(S)$ is surjective, for each $\beta_{S} \in \mathrm{NE}(S)$ represented by a curve $C=\sum_{j} n_{j} C_{j}$, the determination of $\bar{\psi}_{*}^{-1}\left(\beta_{S}\right)$ corresponds to the determination of $\mathrm{NE}\left(P\left(V_{C_{j}}\right)\right)$ for all $j$. Therefore by Lemma 3.1, the minimal lift with respect to this curve decomposition is given by

$$
\beta^{P}:=\sum_{j} n_{j}\left(\bar{\psi}^{*}\left[C_{j}\right] \cdot H_{r}-\mu_{C_{j}} \ell\right)=\beta_{S}-\mu_{\beta_{S}} \ell,
$$

with $\mu_{C_{j}}=\max _{i}\left(C_{j} \cdot L_{i}\right)$ and $\mu=\mu_{\beta_{S}}:=\sum_{j} n_{j} \mu_{C_{j}}$. As before we identify the canonical lift $\bar{\psi}^{*} \beta_{S} \cdot H_{r}$ with $\beta_{S}$. Thus the crucial part is to determine the case of primitive classes. The general case follows from the primitive case by additivity. When there is more than one way to decompose into primitive classes, the minimal lift is obtained by taking the minimal one. Notice that further decomposition leads to a smaller (or equal) lift. Also, there could be more than one minimal lift coming from different (non-comparable) primitive decompositions.

Now we apply the above results to study the effective and $\mathscr{F}$-effective curve classes under the local split ordinary flop $f: X \rightarrow X^{\prime}$ of type $\left(S, F, F^{\prime}\right)$. Fixing a primitive curve class $\beta_{S} \in \operatorname{NE}(S)$, we define

$$
\mu_{i}:=\left(\beta_{S} \cdot L_{i}\right), \quad \mu_{i}^{\prime}:=\left(\beta_{S} \cdot L_{i}^{\prime}\right)
$$

Let $\mu=\max \mu_{i}$ and $\mu^{\prime}=\max \mu_{i}^{\prime}$. Then by working on an irreducible representation curve $C$ of $\beta_{S}$, we get by Lemma 3.1

$$
\begin{aligned}
\mathrm{NE}(Z)_{\beta_{S}} & =\left(\beta_{S}-\mu \ell\right)+\mathbb{Z}_{\geqslant 0} \ell \equiv \beta_{Z}+\mathbb{Z}_{\geqslant 0} \ell, \\
\mathrm{NE}\left(Z^{\prime}\right)_{\beta_{S}} & =\left(\beta_{S}-\mu^{\prime} \ell^{\prime}\right)+\mathbb{Z}_{\geqslant 0} \ell^{\prime} \equiv \beta_{Z^{\prime}}+\mathbb{Z}_{\geqslant 0} \ell^{\prime} .
\end{aligned}
$$

Now we consider the further lifts of the primitive elements $\beta_{Z}$ and $\beta_{Z^{\prime}}$ to $X$. The bundle $N \oplus \mathscr{O}$ is of split type with Chern roots $-h+L_{i}^{\prime}$ and 0 for $i=0, \ldots, r$. On $\beta_{Z}$ they take on values

$$
\mu+\mu_{i}^{\prime} \quad(i=0, \ldots, r) \quad \text { and } \quad 0 .
$$

To determine the minimal lift of $\beta_{Z}$ in $X$, we separate it into two cases.

Case (1): $\mu+\mu^{\prime}>0$. The greatest number in (3.1) is $\mu+\mu^{\prime}$ and

$$
\mathrm{NE}(X)_{\beta_{Z}}=\left(\beta_{Z}-\left(\mu+\mu^{\prime}\right) \gamma\right)+\mathbb{Z}_{\geqslant 0} \gamma
$$

Case (2): $\mu+\mu^{\prime} \leqslant 0$. The greatest number in (3.1) is 0 and

$$
\mathrm{NE}(X)_{\beta_{Z}}=\beta_{Z}+\mathbb{Z}_{\geqslant 0} \gamma
$$

To summarize, we have the following.

Lemma 3.2. Given a primitive class $\beta_{S} \in \mathrm{NE}(S)$, we have $\beta=\beta_{S}+d \ell+d_{2} \gamma \in \mathrm{NE}(X)$ if and only if

$$
d \geqslant-\mu \quad \text { and } \quad d_{2} \geqslant-\nu
$$

where $\nu=\max \left\{\mu+\mu^{\prime}, 0\right\}$.

Remark 3.3. For the general case $\beta_{S}=\sum_{j} n_{j}\left[C_{j}\right]$, the constants $\mu$ and $\nu$ are replaced by

$$
\mu=\mu_{\beta_{S}}:=\sum_{j} n_{j} \mu_{C_{j}} \quad \text { and } \quad \nu=\nu_{\beta_{S}}:=\sum_{j} n_{j} \max \left\{\mu_{C_{j}}+\mu_{C_{j}^{\prime}}, 0\right\} .
$$




\section{INVARIANCE OF QUANTUM RINGS II}

Thus a geometric minimal lift $\beta_{S}^{X} \in \mathrm{NE}(X)$ for $\beta_{S} \in \mathrm{NE}(S)$, with respect to the given primitive decomposition is

$$
\beta_{S}^{X}=\beta_{S}-\mu \ell-\nu \gamma .
$$

(If $\mu_{C_{j}}+\mu_{C_{j}}^{\prime} \geqslant 0$ for all $j$, then $\nu=\mu+\mu^{\prime}$.)

The geometric minimal lifts describe $\mathrm{NE}(X)$. We will however only need a "generic lift" ( $I$-minimal lift in Definition 3.7) in the study of GW invariants.

Definition 3.4. A class $\beta \in N_{1}(X)$ is $\mathscr{F}$-effective if $\beta \in \mathrm{NE}(X)$ and $\mathscr{F} \beta \in \mathrm{NE}\left(X^{\prime}\right)$.

Proposition 3.5. Let $\beta_{S} \in \mathrm{NE}(S)$ be primitive. A class $\beta \in \mathrm{NE}(X)$ over $\beta_{S}$ is $\mathscr{F}$-effective if and only if

$$
d+\mu \geqslant 0 \quad \text { and } \quad d_{2}-d+\mu^{\prime} \geqslant 0 .
$$

Proof. Let $\beta=\beta_{S}+d \ell+d_{2} \gamma$, then $\mathscr{F} \beta=\beta_{S}-d \ell^{\prime}+d_{2}\left(\gamma^{\prime}+\ell^{\prime}\right)=\beta_{S}+\left(d_{2}-d\right) \ell^{\prime}+d_{2} \gamma=$ : $\beta_{S}+d^{\prime} \ell^{\prime}+d_{2}^{\prime} \gamma^{\prime}$. It is clear that $\beta$ being $\mathscr{F}$-effective implies both inequalities. Conversely, the two inequalities imply

$$
d_{2} \geqslant d-\mu^{\prime} \geqslant-\left(\mu+\mu^{\prime}\right) \geqslant-\nu,
$$

hence $\beta \in \mathrm{NE}(X)$. Similarly, $\mathscr{F} \beta \in \mathrm{NE}\left(X^{\prime}\right)$.

\subsection{Symmetry for $I$}

For $F=\bigoplus_{i=0}^{r} L_{i}$ and $F^{\prime}=\bigoplus_{i=0}^{r} L_{i}^{\prime}$, the Chern polynomials for $F$ and $N \oplus \mathscr{O}$ take on the form

$$
f_{F}=\prod a_{i}:=\prod\left(h+L_{i}\right), \quad f_{N \oplus \mathscr{O}}=b_{r+1} \prod b_{i}:=\xi \prod\left(\xi-h+L_{i}^{\prime}\right) .
$$

For $\beta=\beta_{S}+d \ell+d_{2} \gamma$, we set $\mu_{i}:=\left(L_{i} \cdot \beta_{S}\right)$ and $\mu_{i}^{\prime}:=\left(L_{i}^{\prime} \cdot \beta_{S}\right)$. Then for $i=0, \ldots, r$ we have $\left(a_{i} \cdot \beta\right)=d+\mu_{i},\left(b_{i} \cdot \beta\right)=d_{2}-d+\mu_{i}^{\prime}$ and $\left(b_{r+1} \cdot \beta\right)=d_{2}$. Let

$$
\lambda_{\beta}=\left(c_{1}(X / S) \cdot \beta\right)=\left(c_{1}(F)+c_{1}\left(F^{\prime}\right)\right) \cdot \beta_{S}+(r+2) d_{2} .
$$

The relative $I$-factor is given by

$$
I_{\beta}^{X / S}:=\frac{1}{z^{\lambda_{\beta}}} \frac{\Gamma(1+\xi / z)}{\Gamma\left(1+\xi / z+d_{2}\right)} \prod_{i=0}^{r} \frac{\Gamma\left(1+a_{i} / z\right)}{\Gamma\left(1+a_{i} / z+\mu_{i}+d\right)} \frac{\Gamma\left(1+b_{i} / z\right)}{\Gamma\left(1+b_{i} / z+\mu_{i}^{\prime}+d_{2}-d\right)},
$$

and the hypergeometric modification of $\bar{p}: X \rightarrow S$ is

$$
I=I\left(D, \bar{t} ; z, z^{-1}\right)=\sum_{\beta \in \mathrm{NE}(X)} q^{\beta} e^{D / z+(D \cdot \beta)} I_{\beta}^{X / S} J_{\beta_{S}}^{S}(\bar{t}),
$$

where $D=t^{1} h+t^{2} \xi$ is the fiber divisor and $\bar{t} \in H(S)$.

In more explicit terms, for a split projective bundle $\bar{\psi}: P=P(V) \rightarrow S$, the relative $I$-factor equals

$$
\begin{gathered}
I_{\beta}^{P / S}:=\prod_{i=0}^{r} \prod_{m=1}^{\beta \cdot\left(h+L_{i}\right)}\left(h+L_{i}+m z\right)^{-1}, \\
I_{\beta}^{P / S}:=\prod_{i=0}^{r}\left(1 / \prod_{m=1}^{\beta \cdot\left(h+L_{i}\right)}\left(h+L_{i}+m z\right)\right),
\end{gathered}
$$


where the product in $m \in \mathbb{Z}$ is directed in the sense that

$$
\prod_{m=1}^{s}:=\prod_{m=-\infty}^{s} / \prod_{m=-\infty}^{0}
$$

Thus for each $i$ with $\beta \cdot\left(h+L_{i}\right) \leqslant-1$, the corresponding subfactor is understood as occurring in the numerator; furthermore, the numerator must contain the factor $h+L_{i}$ corresponding to $m=0$. In general, $I$ is viewed as a Laurent series in $z^{-1}$ with cohomology-valued coefficients. By the dimension constraint it in fact has only finite terms.

Remark 3.6. The relative factor comes from the equivariant Euler class of

$$
H^{0}\left(C,\left.T_{P / S}\right|_{C}\right)-H^{1}\left(C,\left.T_{P / S}\right|_{C}\right)
$$

at the moduli point $\left[C \cong P^{1} \rightarrow X\right]$.

Definition 3.7 ( $I$-minimal lift). Introduce

$$
\mu_{\beta_{S}}^{I}:=\max _{i}\left\{\beta_{S} \cdot L_{i}\right\}, \quad \mu_{\beta_{S}}^{\prime I}:=\max _{i}\left\{\beta_{S} \cdot L_{i}^{\prime}\right\}
$$

and

$$
\nu_{\beta_{S}}^{I}=\max \left\{\mu_{\beta_{S}}^{I}+\mu_{\beta_{S}}^{\prime I}, 0\right\} \geqslant 0 .
$$

Define the $I$-minimal lift of $\beta_{S}$ to be

$$
\beta_{S}^{I}:=\beta_{S}-\mu_{\beta_{S}}^{I} \ell-\nu_{\beta_{S}}^{I} \gamma \in \mathrm{NE}(X)
$$

where $\beta_{S} \in \mathrm{NE}(X)$ is the canonical lift such that $h \cdot \beta_{S}=0=\xi \cdot \beta_{S}$.

Clearly, $\beta_{S}^{I}$ is an effective class in $\mathrm{NE}(X)$, as $\mu_{\beta_{S}}^{I} \leqslant \mu_{\beta_{S}}$ and $\nu_{\beta_{S}}^{I} \leqslant \nu_{\beta_{S}}$. When the inequality is strict, the $I$-minimal lift is more effective than any geometric minimal lift. Nevertheless, it is uniquely defined and we will show that it encodes the information of the hypergeometric modification.

Definition 3.8. Define $\beta$ to be $I$-effective, denoted by $\beta \in \operatorname{NE}^{I}(X)$, if

$$
d \geqslant-\mu_{\beta_{S}}^{I} \quad \text { and } \quad d_{2} \geqslant-\nu_{\beta_{S}}^{I} .
$$

It is called $\mathscr{F} I$-effective if $\beta$ is $I$-effective and $\mathscr{F} \beta$ is $I^{\prime}$-effective. By the same proof as that of Proposition 3.5, this is equivalent to

$$
d+\mu_{\beta_{S}}^{I} \geqslant 0 \quad \text { and } \quad d_{2}-d+\mu_{\beta_{S}}^{I} \geqslant 0
$$

Lemma 3.9 (Vanishing lemma). If $\bar{\psi}_{*} \beta \in \mathrm{NE}(S)$ but $\beta \notin \mathrm{NE}(P)$, then $I_{\beta}^{P / S}=0$. In fact, the vanishing statement holds for any $\beta=\beta_{S}+d \ell$ with $d<-\mu_{\beta_{S}}^{I}$.

Proof. We have $\beta \cdot\left(h+L_{i}\right)=d+\mu_{i} \leqslant d+\mu_{\beta_{S}}^{I}<0$ for all $i$. This implies $I_{\beta}^{P / S}=0$ since it contains the Chern polynomial factor $\prod_{i}\left(h+L_{i}\right)=0$ in the numerator.

Now $I_{\beta}^{X / S} \equiv I_{\beta}^{Z / S} I_{\beta}^{X / Z}$ is given by

$$
\prod_{i=0}^{r} \prod_{m=1}^{\beta \cdot a_{i}}\left(a_{i}+m z\right)^{-1} \prod_{i=0}^{r} \prod_{m=1}^{\beta \cdot b_{i}}\left(b_{i}+m z\right)^{-1} \prod_{m=1}^{\beta \cdot \xi}(\xi+m z)^{-1}=: A_{\beta} B_{\beta} C_{\beta} .
$$

Although (3.10) makes sense for any $\beta \in N_{1}(X)$, we have the following. 


\section{INVARIANCE OF QUANTUM RINGS II}

Lemma 3.10. The $I$-factor $I_{\beta}^{X / S}$ is non-trivial only if $\beta \in \mathrm{NE}^{I}(X)$.

Proof. Indeed, if $\beta_{S} \in \mathrm{NE}(S)$ but $\beta \notin \mathrm{NE}^{I}(X)$, then either $d<-\mu_{\beta_{S}}^{I}$ and $A_{\beta}=0$ by Lemma 3.9, or $d \geqslant-\mu_{\beta_{S}}^{I}$ and we must have $d_{2}<-\nu_{\beta_{S}}^{I} \leqslant 0$ and all factors in $B_{\beta}$ appear in the numerator:

$$
d_{2}-d+\mu_{i}^{\prime} \leqslant d_{2}+\mu_{\beta_{S}}^{I}+\mu_{\beta_{S}}^{I} \leqslant d_{2}+\nu_{\beta_{S}}^{I}<0 .
$$

In particular, $B_{\beta} C_{\beta}$ contains the Chern polynomial $f_{N \oplus \mathscr{O}}=0$.

Remark 3.11. In view of Lemma 3.2, if $\beta$ is a primitive class, then $\beta \in \mathrm{NE}^{I}(X)$ if and only if $\beta \in \mathrm{NE}(X)$. Hence the condition $\beta \in \mathrm{NE}^{I}(X)$ is the "effective condition that $\beta$ behaves as a primitive class." One way to think about this is that the localization calculation of the $I$-factor is performed on the main component of the stable map moduli space where $\beta$ is represented by a smooth rational curve.

As far as $I$ is concerned, the $I$-effective class plays the role of effective class. However, one needs to be careful that the converse of Lemma 3.10 is not true: If $\beta$ is $I$-effective, it is still possible to have $I_{\beta}^{X / S}=0$.

The expression (3.10) agrees with (3.5) by taking out the $z$-factor with $m$. The total factor is clearly

$$
z^{-\left(\sum_{i=0}^{r} a_{i}+\sum_{i=0}^{r+1} b_{i}\right) \cdot \beta}=z^{-c_{1}(X / S) \cdot \beta} .
$$

Similarly, for $\beta^{\prime} \in \mathrm{NE}\left(X^{\prime}\right)$, the $I$-factor $I_{\beta^{\prime}}^{X^{\prime} / S} \equiv I_{\beta^{\prime}}^{Z^{\prime} / S} I_{\beta^{\prime}}^{X^{\prime} / Z^{\prime}}$ is given by

$$
\prod_{i=0}^{r} \prod_{m=1}^{\beta^{\prime} \cdot a_{i}^{\prime}}\left(a_{i}^{\prime}+m z\right)^{-1} \prod_{i=0}^{r} \prod_{m=1}^{\beta^{\prime} \cdot b_{i}^{\prime}}\left(b_{i}^{\prime}+m z\right)^{-1} \prod_{m=1}^{\beta^{\prime} \cdot \xi^{\prime}}\left(\xi^{\prime}+m z\right)^{-1}=: A_{\beta^{\prime}}^{\prime} B_{\beta^{\prime}}^{\prime} C_{\beta^{\prime}}^{\prime} .
$$

Here $a_{i}^{\prime}=h^{\prime}+L_{i}^{\prime}=\mathscr{F} b_{i}$ and $b_{i}^{\prime}=\xi^{\prime}-h^{\prime}+L_{i}=\mathscr{F} a_{i}$.

By the invariance of the Poincaré pairing, $\left(\beta \cdot a_{i}\right)=d+\mu_{i}=\left(\mathscr{F} \beta \cdot b_{i}^{\prime}\right)$ and $\left(\beta \cdot b_{i}\right)=d_{2}-d+\mu_{i}^{\prime}=$ $\left(\mathscr{F} \beta \cdot a_{i}^{\prime}\right)$, and it is clear that all the linear subfactors in $I_{\beta}^{X / S}$ and $I_{\mathscr{F} \beta}^{X^{\prime} / S}$ correspond perfectly under $A_{\beta} \mapsto B_{\mathscr{F} \beta}^{\prime}, B_{\beta} \mapsto A_{\mathscr{F} \beta}^{\prime}$ and $C_{\beta} \mapsto C_{\mathscr{F} \beta}^{\prime}$.

However, since the cup product is not preserved under $\mathscr{F}$, in general $\mathscr{F} I_{\beta} \neq I_{\mathscr{F} \beta}^{\prime}$. Clearly, any direct comparison of $I_{\beta}$ and $I_{\mathscr{F} \beta}^{\prime}$ (without analytic continuations) can make sense only if $\beta$ is $\mathscr{F} I$-effective. This is the case if the $\left(\beta \cdot a_{i}\right)$ and $\left(\beta \cdot b_{i}\right)$, respectively, are not all negative. Namely, $A_{\beta}$ and $B_{\beta}$ both contain factors in the denominator.

LEMma 3.12 (Naive quasi-linearity). (1) $\mathscr{F} I_{\beta} \cdot \xi=I_{\mathscr{F} \beta}^{\prime} \cdot \xi^{\prime}$.

(2) If $d_{2}:=\beta . \xi<0$, then $\mathscr{F} I_{\beta}=I_{\mathscr{F} \beta}^{\prime}$.

The expressions in statements (1) or (2) are non-trivial only if $\beta$ is $\mathscr{F} I$-effective.

Proof. Statement (1) follows from the fact that $f: X \rightarrow X^{\prime}$ is an isomorphism over the infinity divisors $E \cong E$. For statement (2), notice that since $d_{2}<0$, the factor $C_{\beta}$ contains $\xi$ in the numerator corresponding to $m=0$. Similarly, $C_{\mathscr{F} \beta}^{\prime}$ contains $\xi^{\prime}$ in the numerator. Hence, statement (2) follows for the same reason as statement (1). The last statement follows from Lemma 3.10.

\subsection{The Picard-Fuchs system}

Now, we return to the BF/GMT constructed in Theorem 2.10 and multiply it by the infinity divisor $\xi$ :

$$
J^{X}(\tau(\hat{t})) \cdot \xi=P(z) I^{X}(\hat{t}) \cdot \xi
$$


By Proposition 2.11 and Lemma 3.12, we need to show the $\mathscr{F}$-invariance for $P(z)$ and $\tau(\hat{t})$ in order to establish the general analytic continuation.

The very first evidence for this is that, as in the case of classical hypergeometric series, $I^{X}$ and $I^{X^{\prime}}$ are solutions of certain Picard-Fuchs systems which turn out to be $\mathscr{F}$-compatible.

Proposition 3.13 (Picard-Fuchs system on $X$ ). We have $\square_{\ell} I^{X}=0$ and $\square_{\gamma} I^{X}=0$, where

$$
\square_{\ell}=\prod_{j=0}^{r} z \partial_{a_{j}}-q^{\ell} e^{t^{1}} \prod_{j=0}^{r} z \partial_{b_{j}}, \quad \square_{\gamma}=z \partial_{\xi} \prod_{j=0}^{r} z \partial_{b_{j}}-q^{\gamma} e^{t^{2}} .
$$

Recall that $t^{1}$ and $t^{2}$ are the dual coordinates of $h$ and $\xi$, respectively. Here we use $\partial_{v}$ to denote the directional derivative in $v$. Thus if $v=\sum v^{i} T_{i} \in H^{2}$, then $\partial_{v}=\sum v^{i} \partial_{t^{i}}$.

Proof. By extracting all the divisor variables $D=t^{1} h+t^{2} \xi$ and $\bar{t}_{1} \in H^{2}(S)$ from $I^{X}$ (where $\left.\bar{t}=\bar{t}_{1}+\bar{t}_{2}\right)$, we get

$$
I^{X}=\sum_{\beta \in \mathrm{NE}(X)} q^{\beta} e^{\left(D+\bar{t}_{1}\right) / z+\left(D+\bar{t}_{1}\right) \cdot \beta} I_{\beta}^{X / S} J_{\beta_{S}}^{S}\left(\bar{t}_{2}\right) .
$$

It is clear that $z \partial_{v}$ produces the factor $v+z(v \cdot \beta)$ for $v \in H^{2}$. From (3.10), we deduce that $\prod_{j} z \partial_{a_{j}}$ modifies the $A_{\beta} B_{\beta} C_{\beta}$-factor to

$$
\prod_{j=0}^{r} \prod_{m=1}^{\beta \cdot a_{j}-1}\left(a_{j}+m z\right)^{-1} B_{\beta} C_{\beta}=A_{\beta-\ell} B_{\beta-\ell} \prod_{j=0}^{r}\left(b_{j}+z(\beta-\ell) \cdot b_{j}\right) C_{\beta-\ell}
$$

$\left(\right.$ since $\beta \cdot a_{j}-1=(\beta-\ell) \cdot a_{j},(\beta-\ell) \cdot b_{j}=\beta \cdot b_{j}+1$ and $\left.(\beta-\ell) \cdot \xi=\beta \cdot \xi\right)$.

Clearly it equals the corresponding term from $q^{\ell} e^{t^{1}} \prod_{j} z \partial_{b_{j}} I^{X}$ unless $\beta-\ell$ is not effective. But in that case the term is itself zero since $A_{\beta-\ell}=0$ by Lemma 3.9.

The proof for $\square_{\gamma} I^{X}=0$ is similar and is thus omitted.

Similarly, $I^{X^{\prime}}$ is a solution to

$$
\square_{\ell^{\prime}}=\prod_{j=0}^{r} z \partial_{a_{j}^{\prime}}-q^{\ell^{\prime}} e^{-t^{1}} \prod_{j=0}^{r} z \partial_{b_{j}^{\prime}}, \quad \square_{\gamma^{\prime}}=z \partial_{\xi^{\prime}} \prod_{j=0}^{r} z \partial_{b_{j}^{\prime}}-q^{\gamma^{\prime}} e^{t^{2}+t^{1}},
$$

where the dual coordinates of $h^{\prime}$ and $\xi^{\prime}$ are $-t^{1}$ and $t^{2}+t^{1}$, respectively (since $\mathscr{F}\left(t^{1} h+t^{2} \xi\right)=$ $\left.t^{1}\left(\xi^{\prime}-h^{\prime}\right)+t^{2} \xi^{\prime}=\left(-t^{1}\right) h^{\prime}+\left(t^{2}+t^{1}\right) \xi^{\prime}\right)$.

Proposition 3.14. $\mathscr{F}\left\langle\square_{\ell}^{X}, \square_{\gamma}^{X}\right\rangle \cong\left\langle\square_{\ell^{\prime}}^{X^{\prime}}, \square_{\gamma^{\prime}}^{X^{\prime}}\right\rangle$.

Proof. It is clear that

$$
\mathscr{F} \square_{\ell}=-q^{-\ell^{\prime}} e^{t^{1}} \square_{\ell^{\prime}}
$$

and

$$
\mathscr{F} \square_{\gamma}=z \partial_{\xi^{\prime}} \prod_{j=0}^{r} z \partial_{a_{j}^{\prime}}-q^{\gamma^{\prime}+\ell^{\prime}} e^{t^{2}}=z \partial_{\xi^{\prime}} \square_{\ell^{\prime}}+q^{\ell^{\prime}} e^{-t^{1}} \square_{\gamma^{\prime}} .
$$

Namely, the Picard-Fuchs systems on $X$ and $X^{\prime}$ are indeed equivalent under $\mathscr{F}$. Moreover, both $I=I^{X}$ and $I^{\prime}=I^{X^{\prime}}$ satisfy this system, but in different coordinate charts (of the Kähler moduli space) " $\left|q^{\ell}\right|<1$ " and " $\left|q^{\ell}\right|>1$ ", respectively.

We do not expect $I$ and $I^{\prime}$ to be the same solution under analytic continuation in general. In fact, they are not in some examples. We know this is not true for $J$ and $J^{\prime}$ since the general 


\section{INVARIANCE OF QUANTUM RINGS II}

descendent invariants are not $\mathscr{F}$-invariant. Nevertheless, it turns out that $P(z)$ and $\tau(\hat{t})$ are correct objects to admit $\mathscr{F}$-invariance.

Lemma 3.15. Modulo $q^{\beta_{S}}, \beta_{S} \in \mathrm{NE}(S)$ and $\gamma$, we have $P(z) \equiv 1$ and $\tau(\hat{t}) \equiv \hat{t}$.

Proof. One simply notices that in the proof of Theorem 2.10, to construct $P(z)$ the induction can be performed on $[\beta]=\left(\beta_{S}, d_{2}\right) \in W$, as in [LLW16, Section 4.2], by removing the whole series in $q^{\ell}$ with the same top non-negative $z$-power one at a time. For the initial step $[\beta]=0$ and $J^{S}([\beta]=0)=e^{\bar{t} / z}$, from (3.10) we have extremal ray contributions:

$$
I_{[\beta]=0}=e^{\hat{t} / z}\left(1+O\left(1 / z^{r+1}\right)\right) .
$$

As there are no non-negative $z$-powers besides 1 , later inductive steps will also create only higherorder $q^{[\beta]}$ with respect to $W$, hence the result follows.

Remark 3.16. By a virtual dimension count and (2.1), the $J$-function is weighted homogeneous of degree 0 in the following weights $|\cdot|$ : we set $\left|T_{\mu}\right|$ to be its Chow degree, $\left|t^{\mu}\right|=1-\left|T_{\mu}\right|$ and $\left|q^{\beta}\right|=\left(c_{1}(X) \cdot \beta\right)$, and finally $|\psi|=|z|=1$. This is usually expressed as: the Frobenius manifold $(\mathrm{QH}(X), *)$ is conformal with respect to the Euler vector field

$$
E=\sum\left(1-\left|T_{\mu}\right|\right) t^{\mu} \partial_{\mu}+c_{1}(X) \in \Gamma(T H) .
$$

For the hypergeometric modification $I$, the base $J^{S}$ has degree zero with $\left|q^{\beta_{S}}\right|=\left(c_{1}(S) \cdot \beta_{S}\right)$. But when $\beta_{S}$ is viewed as an object in $X$, the weight increases by $\left(c_{1}(X / S) \cdot \beta_{S}\right)$. This cancels with the weight of the factor $I^{X / S} q^{\beta-\beta_{S}}$, which is

$$
\begin{aligned}
-c_{1}(X / S) \cdot \beta+c_{1}(X) \cdot \beta-c_{1}(X) \cdot \beta_{S} & =c_{1}(S) \cdot \beta-c_{1}(X) \cdot \beta_{S} \\
& =-c_{1}(X / S) \cdot \beta_{S} .
\end{aligned}
$$

Hence $I$ is also homogeneous of degree zero.

\section{Extension of quantum $\mathscr{D}$-modules via the quantum Leray-Hirsch theorem}

In this section we will complete the proof of the main theorem (Theorem 1.3) on the invariance of quantum rings under ordinary flops of split type. Proposition 3.14 guarantees the $\mathscr{F}$-invariance of the Picard-Fuchs systems (in the fiber directions). In order to construct the $\mathscr{D}$-module $\mathscr{M}_{I}=\mathscr{D} I$, we will need to find the derivatives in the general base directions. This will be accomplished by a lifting of the QDE on the base $S$. Putting these together, we will show that they generate enough (correct) equations for $\mathscr{M}_{I}^{X}$. This is referred to as the quantum Leray-Hirsch theorem, which is the content of Theorem 1.2 (a combination of Theorems 4.6, 4.8 and 4.10).

To obtain the (true) quantum $\mathscr{D}$-module $\mathscr{M}_{J}^{X}$ (on a sufficiently large Zariski-closed subset given by the image of $\tau(\hat{t})$ ), we apply the Birkhoff factorization on $\mathscr{M}_{I}^{X}$. We specifically choose a way to perform the BF such that the $\mathscr{F}$-invariance can be checked more naturally.

Before proceeding to the first step, let us lay out the notation and conventions for this section.

Notation 4.1. We use $\bar{\beta} \in \mathrm{NE}(S), \bar{t} \in H(S)$ etc. to denote objects in $S$. When they are viewed as objects in $X$, however, $\bar{\beta}$ means the canonical lift and $\bar{t}$ means the pullback $\bar{p}^{*}: H(S) \rightarrow H(X)$.

For a basis $\left\{\bar{T}_{i}\right\}$ of $H(S)$, denote by $\bar{t}=\sum \bar{t}^{i} \bar{T}_{i}$ a general element in $H(S)$. When $\bar{T}_{i}$ is considered as an element in $H(X)$, we sometimes abuse the notation by setting $T_{i}:=\bar{T}_{i}$.

Given a basis $\left\{\bar{T}_{i}\right\}$ of $H(S)$, we use the following canonical basis for $H(X)$ :

$$
\left\{T_{\mathbf{e}}=\bar{T}_{i} h^{l} \xi^{m} \mid 0 \leqslant l \leqslant r, 0 \leqslant m \leqslant r+1\right\} .
$$


A general element in $H(X)$ is denoted by $t=\sum t^{\mathbf{e}} T_{\mathbf{e}}$. The index set of the canonical basis is denoted by $\Lambda^{+}$.

By abusing the notation, if $T_{\mathbf{e}}=\bar{T}_{i}$ (that is, $l=m=0$ ), we set $t^{\mathbf{e}}=t^{i}=\bar{t}^{i}$. Similarly, we set $t^{\mathbf{e}}=t^{1}$ for $T_{\mathbf{e}}=h$ and $t^{\mathbf{e}}=t^{2}$ for $T_{\mathbf{e}}=\xi$. That is, we reserve the indices 0,1 and 2 for $1, h$ and $\xi$, respectively.

On $H\left(X^{\prime}\right)$ the canonical basis is chosen to be

$$
\left\{T_{\mathbf{e}}^{\prime}:=\mathscr{F} T_{\mathbf{e}}=\bar{T}_{i}\left(\xi^{\prime}-h^{\prime}\right)^{l} \xi^{\prime m}\right\},
$$

so that it shares the same coordinate system as $H(X)$ :

$$
t=\sum_{\mathbf{e}} t^{\mathbf{e}} T_{\mathbf{e}} \mapsto \mathscr{F} t=\sum_{\mathbf{e}} t^{\mathbf{e}} \mathscr{F} T_{\mathbf{e}}=\sum_{\mathbf{e}} t^{\mathbf{e}} T_{\mathbf{e}}^{\prime}
$$

\subsection{I-lift of the Dubrovin connection}

Let the quantum differential equation of $\mathrm{QH}(S)$ be given by

$$
z \partial_{i} z \partial_{j} J^{S}(\bar{t})=\sum_{k} \bar{C}_{i j}^{k}(\bar{t}, \bar{q}) z \partial_{k} J^{S}(\bar{t}) .
$$

If we write $\bar{C}_{i j}^{k}(\bar{t}, \bar{q})=\sum \bar{C}_{i j, \bar{\beta}}^{k}(\bar{t}) q^{\bar{\beta}}$, then the effect on the $\bar{\beta}$-components reads as

$$
z \partial_{i} z \partial_{j} J_{\bar{\beta}}^{S}=\sum_{k, \bar{\beta}_{1}} \bar{C}_{i j, \bar{\beta}_{1}}^{k} z \partial_{k} J_{\bar{\beta}-\bar{\beta}_{1}}^{S} .
$$

Now we lift the equation to $X$. In the following, for a curve class $\bar{\beta} \in \mathrm{NE}(S)$, its $I$-minimal lift in $\mathrm{NE}(X)$ is denoted by $\bar{\beta}^{I}$. We compute

$$
\begin{aligned}
z \partial_{i} z \partial_{j} I & =\sum_{\beta} q^{\beta} e^{D / z+(D \cdot \beta)} I_{\beta}^{X / S} z \partial_{i} z \partial_{j} J_{\bar{\beta}}^{S} \\
& =\sum_{k, \beta, \bar{\beta}_{1}} q^{\beta} e^{D / z+(D \cdot \beta)} I_{\beta}^{X / S} \bar{C}_{i j, \bar{\beta}_{1}}^{k} z \partial_{k} J_{\bar{\beta}-\bar{\beta}_{1}}^{S} \\
& =\sum_{k, \bar{\beta}_{1}} q^{\bar{\beta}_{1}^{I}} e^{D \cdot \bar{\beta}_{1}^{I}} \bar{C}_{i j, \bar{\beta}_{1}}^{k} z \partial_{k} \sum_{\beta} q^{\beta-\bar{\beta}_{1}^{I}} e^{D / z+D \cdot\left(\beta-\bar{\beta}_{1}^{I}\right)} I_{\beta}^{X / S} J_{\bar{\beta}_{-}-\bar{\beta}_{1}} .
\end{aligned}
$$

The terms in last sum are non-trivial only if $\bar{\beta}-\bar{\beta}_{1} \in \mathrm{NE}(S)$. However, in this presentation it is not a priori guaranteed that $\beta-\bar{\beta}_{1}^{I}$ is $I$-effective. (Hence, there might be some vanishing terms in the presentation.)

In order to obtain the right-hand side as an operator acting on $I$, we will seek to "transform" terms of the form

to terms of the form

$$
e^{D / z+D \cdot\left(\beta-\bar{\beta}_{1}^{I}\right)} I_{\beta}^{X / S} J J_{\bar{\beta}-\bar{\beta}_{1}}^{S}
$$

$$
e^{D / z+D \cdot\left(\beta-\bar{\beta}_{1}^{I}\right)} I_{\beta-\bar{\beta}_{1}^{I}}^{X / S} J_{\bar{\beta}-\bar{\beta}_{1}}^{S} .
$$

This can be achieved by differentiating the right-hand side judiciously and will be explained below.

As a first step, we will show that $I_{\beta}^{X / S}=0$ if $\beta-\bar{\beta}_{1}^{I} \notin \mathrm{NE}^{I}(X)$ and $\bar{\beta}-\bar{\beta}_{1} \in \mathrm{NE}(S)$.

Definition 4.2. For any 1-cycle $\beta \in A_{1}(X)$, effective or not, we define

$$
n_{i}(\beta):=-\beta \cdot\left(h+L_{i}\right), \quad n_{i}^{\prime}(\beta):=-\beta \cdot\left(\xi-h+L_{i}^{\prime}\right), \quad n_{r+1}^{\prime}(\beta):=-\beta \cdot \xi,
$$




\section{INVARIANCE OF QUANTUM RINGS II}

where $0 \leqslant i \leqslant r$.

Lemma 4.3. For $\bar{\beta} \in \mathrm{NE}(S)$, the $I$-minimal lift $\bar{\beta}^{I} \in \mathrm{NE}(X)$ satisfies $n_{i}\left(\bar{\beta}^{I}\right) \geqslant 0$ and $n_{i}^{\prime}\left(\bar{\beta}^{I}\right) \geqslant 0$ for all $i$.

Proof. During the proof, the superscript $I$ is omitted for simplicity.

By definition,

$$
n_{i}=-\bar{\beta}^{I} \cdot\left(h+L_{i}\right)=\mu-\mu_{i} \geqslant 0 .
$$

Similarly, for $0 \leqslant i \leqslant r$,

$$
n_{i}^{\prime}=-\bar{\beta}^{I} \cdot\left(\xi-h+L_{i}^{\prime}\right)=\max \left\{\mu+\mu^{\prime}, 0\right\}-\mu-\mu_{i}^{\prime} .
$$

If $\mu+\mu^{\prime} \geqslant 0$, we have

$$
n_{i}^{\prime}=\mu^{\prime}-\mu_{i}^{\prime} \geqslant 0
$$

Otherwise, if $\mu+\mu^{\prime}<0$, then we get

$$
n_{i}^{\prime}=0-\left(\mu+\mu_{i}^{\prime}\right) \geqslant-\left(\mu+\mu^{\prime}\right)>0 .
$$

Finally, for the compactification factor $\mathscr{O}$, we get

$$
n_{r+1}^{\prime}=-\bar{\beta}^{I} \cdot \xi=\max \left\{\mu+\mu^{\prime}, 0\right\} \geqslant 0 .
$$

Let $\beta, \beta^{\prime} \in A_{1}(X)$ be (not necessarily effective) 1-cycles. By definition of the $I$-function, the $\beta$-factor corresponding to $h+L_{i}$ is

$$
A_{i, \beta}=\prod_{m=1}^{\beta \cdot\left(h+L_{i}\right)}\left(h+L_{i}+m z\right)^{-1},
$$

which depends only on the intersection number. Suppose

$$
l_{i}:=\beta^{\prime} \cdot\left(h+L_{i}\right)-\beta \cdot\left(h+L_{i}\right) \geqslant 0,
$$

we then have

$$
A_{i, \beta}=A_{i, \beta^{\prime}} \prod_{m=\beta \cdot\left(h+L_{i}\right)+1}^{\beta^{\prime} \cdot\left(h+L_{i}\right)}\left(h+L_{i}+m z\right) .
$$

We say that $A_{i, \beta}$ is a product of $A_{i, \beta^{\prime}}$ with a (cohomology-valued) factor of length $l_{i}$. The factors corresponding to $\xi-h+L_{i}^{\prime}$ and $\xi$ behave similarly.

Lemma 4.4. Let $\beta \in \mathrm{NE}(X)$, and let $\beta-\bar{\beta}_{1}^{I}$ be an $I$-effective class. Then $I_{\beta}^{X / S}$ is the product of $I_{\beta-\bar{\beta}_{1}^{I}}^{X / S}$ with factors of lengths $n_{i}\left(\bar{\beta}_{1}^{I}\right), n_{i}^{\prime}\left(\bar{\beta}_{1}^{I}\right)$ and $n_{r+1}^{\prime}\left(\bar{\beta}_{1}^{I}\right)$ corresponding to $h+L_{i}, \xi-h+L_{i}^{\prime}$ and $\xi$, respectively.

If $\beta-\bar{\beta}_{1}^{I}$ is not $I$-effective, the conclusion holds in the sense that $I_{\beta}^{X / S}=0$.

Proof. Set $\beta^{\prime}=\beta-\bar{\beta}_{1}^{I}$ in (4.3). The length is

$$
\left(\beta^{\prime}-\beta\right) \cdot\left(h+L_{i}\right)=-\bar{\beta}_{1}^{I} \cdot\left(h+L_{i}\right)=n_{i}\left(\bar{\beta}_{1}^{I}\right) .
$$

The arguments for $\xi-h+L_{i}^{\prime}$ and $\xi$ are similar.

If $\beta-\bar{\beta}_{1}^{I}$ is not $I$-effective, formally $I_{\beta-\bar{\beta}_{1}^{I}}^{X / S}=0$ contains either the Chern polynomial $f_{F}$ or $f_{N \oplus \mathscr{O}}$ in its numerator. Notice that (4.3) holds formally.

This proves the lemma. 
Our next step is to show that the factors in (4.3) can be obtained by introducing certain differential operators acting on $I$.

Definition 4.5. A 1 -cycle $\beta \in A_{1}(X)$ is called admissible if we have $n_{i}(\beta) \geqslant 0, n_{i}^{\prime}(\beta) \geqslant 0$ and $n_{r+1}^{\prime}(\beta) \geqslant 0$. For admissible $\beta$ we define differential operators

$$
\begin{aligned}
D_{\beta}^{A} & :=\prod_{i=0}^{r} \prod_{m=0}^{n_{i}(\beta)-1}\left(z \partial_{h+L_{i}}-m z\right) \\
D_{\beta}^{B} & :=\prod_{i=0}^{r} \prod_{m=0}^{n_{i}^{\prime}(\beta)-1}\left(z \partial_{\xi-h+L_{i}^{\prime}}-m z\right) \\
D_{\beta}^{C} & :=\prod_{m=0}^{n_{r+1}^{\prime}(\beta)-1}\left(z \partial_{\xi}-m z\right) \\
D_{\beta}(z) & :=D_{\beta}^{A} D_{\beta}^{B} D_{\beta}^{C} .
\end{aligned}
$$

Now we are ready to lift the quantum differential equations for $J^{S}$ to equations for $I^{X}$. Theorem 4.6 (I-lift of QDE). The Dubrovin connection on $\mathrm{QH}(S)$ can be lifted to $H(X)$ as

$$
z \partial_{i} z \partial_{j} I=\sum_{k, \bar{\beta}} q^{\bar{\beta}^{*}} e^{D \cdot \bar{\beta}^{*}} \bar{C}_{i j, \bar{\beta}}^{k}(\bar{t}) z \partial_{k} D_{\bar{\beta}^{*}}(z) I,
$$

where $\bar{\beta}^{*} \in A_{1}(X)$ is any admissible lift of $\bar{\beta}$, which in particular implies the well-definedness of the operators $D_{\bar{\beta}^{*}}(z)$.

Furthermore, one can always choose $\bar{\beta}^{*}$ to be effective. An example of an effective lift is the I-minimal lift $\bar{\beta}^{*}=\bar{\beta}^{I}$, which is the only admissible lift if and only if $\mu+\mu^{\prime} \geqslant 0$.

In general, all lifts are related to one another modulo the Picard-Fuchs system generated by $\square_{\ell}$ and $\square_{\gamma}$.

Proof. We apply the calculation in (4.1) with $\bar{\beta}_{1}^{I}$ replaced by a general admissible lift $\bar{\beta}_{1}^{*}$. For $\bar{t}=\bar{t}_{1}+\bar{t}_{2}$, with $\bar{t}_{1}$ the divisor part,

$$
\begin{aligned}
& \sum_{\beta} q^{\beta-\bar{\beta}_{1}^{*}} e^{D / z+D \cdot\left(\beta-\bar{\beta}_{1}^{*}\right)} I_{\beta}^{X / S} J_{\bar{\beta}-\bar{\beta}_{1}}^{S}(\bar{t}) \\
& \quad=\sum_{\beta} D_{\bar{\beta}_{1}^{*}}(z) q^{\beta-\bar{\beta}_{1}^{*}} e^{\left(D+\bar{t}_{1}\right) / z+\left(D+\bar{t}_{1}\right) \cdot\left(\beta-\bar{\beta}_{1}^{*}\right)} I_{\beta-\bar{\beta}_{1}^{*}}^{X / S} J_{\bar{\beta}-\bar{\beta}_{1}}^{S}\left(\bar{t}_{2}\right)=D_{\bar{\beta}_{1}^{*}}(z) I .
\end{aligned}
$$

Now we prove the last statement. Any two (admissible) lifts differ by some $a \ell+b \gamma$, say, $\beta^{\prime \prime}=\beta^{\prime}+a \ell+b \gamma$. Then we have

$$
n_{i}\left(\beta^{\prime \prime}\right)=n_{i}\left(\beta^{\prime}\right)-a, \quad n_{i}^{\prime}\left(\beta^{\prime \prime}\right)=n_{i}^{\prime}\left(\beta^{\prime}\right)+(a-b), \quad n_{r+1}^{\prime}\left(\beta^{\prime \prime}\right)=n_{r+1}^{\prime}\left(\beta^{\prime}\right)-b .
$$

Then it is elementary to see that we may connect $\beta^{\prime}$ to $\beta^{\prime \prime}$ by adding or subtracting $\ell$ or $\gamma$ one at a time, with all the intermediate steps $\beta_{j}^{\prime}$ being admissible. For example, if $a>0, b>0$ and $a-b>0$, then we start by adding $\ell$ up to $j=a-b$ times. Then we iterate the process: adding $\gamma$ followed by adding $\ell$, up to $b$ times. Thus we only have to consider the two cases $(1) \beta^{\prime \prime}=\beta^{\prime}+\ell$ and $(2) \beta^{\prime \prime}=\beta^{\prime}+\gamma$.

For case $(1)$, we get from $(4.5)$ with $(a, b)=(1,0)$ that $n_{i}\left(\beta^{\prime}\right) \geqslant 1$ for all $i$. This implies $D_{\beta^{\prime}}^{A}=D_{\beta^{\prime}}^{A+} D_{0}^{A}$, where $D_{0}^{A}=\prod_{j=0}^{r} z \partial_{a_{j}}$ comes from the product of $m=0$ terms. Since $\square_{\ell} I=0$, 
we compute

$$
D_{\beta^{\prime}}(z) I=D_{\beta^{\prime}}^{B} D_{\beta^{\prime}}^{C} D_{\beta^{\prime}}^{A+} q^{\ell} e^{t^{1}} \prod_{j=0}^{r} z \partial_{b_{j}} I .
$$

Now we move $q^{\ell} e^{t^{1}}$ to the left-hand side of all operators by noticing that

$$
z \partial_{h} e^{t^{1}}=e^{t^{1}}\left(z \partial_{h}+z\right)
$$

in the operator sense. Then (notice that $D_{\beta^{\prime}}^{C}=D_{\beta^{\prime}+\ell}^{C}$ )

$$
D_{\beta^{\prime}}(z) I=q^{\ell} e^{t^{1}} D_{\beta^{\prime}+\ell}^{B+} D_{\beta^{\prime}}^{C} D_{\beta^{\prime}+\ell}^{A} \prod_{j=0}^{r} z \partial_{b_{j}} I=q^{\ell} e^{t^{1}} D_{\beta^{\prime}+\ell}(z) I,
$$

which is the desired factor for $\beta^{\prime \prime}$.

The proof for case (2) is entirely similar, with $\square_{\gamma} I=0$ used instead, and is thus omitted.

The uniqueness statement for $\mu+\mu^{\prime} \geqslant 0$ follows from (4.5) and the observation that $n_{i}\left(\bar{\beta}^{I}\right)=$ $\mu-\mu_{i}$ and $n_{i}^{\prime}\left(\bar{\beta}^{I}\right)=\mu^{\prime}-\mu_{i}^{\prime}$ both attain zero somewhere and there is no room to move around. The proof is complete.

Notice that the lifts of the QDE may not be unique. We will see the importance of such freedom when we discuss the $\mathscr{F}$-invariance property.

\subsection{The quantum Leray-Hirsch theorem}

Definition 4.7. Let $T_{\mathbf{e}}=\bar{T}_{i} h^{l} \xi^{m}$ be an element of the canonical basis of $H(X)$. The naive quantization of $T_{\mathbf{e}}$ is defined as (cf. (2.2) and (2.9))

$$
\hat{T}_{\mathbf{e}}:=\partial^{z \mathbf{e}}=z \partial_{\bar{t}^{i}}\left(z \partial_{t^{1}}\right)^{l}\left(z \partial_{t^{2}}\right)^{m} .
$$

Theorem 4.8 (Quantum Leray-Hirsch). The I-lift (4.4) of quantum differential equations on $S$ and the Picard-Fuchs equations determine a first-order matrix system under the naive quantization $\partial^{z \mathbf{e}}$ of the canonical basis $T_{\mathbf{e}}$ of $H(X)$ :

$$
z \partial_{a}\left(\partial^{z \mathbf{e}} I\right)=\left(\partial^{z \mathbf{e}} I\right) C_{a}(z, q), \quad t^{a} \in\left\{t^{1}, t^{2}, \bar{t}^{i}\right\} .
$$

This system has the property that for any fixed $\bar{\beta} \in \mathrm{NE}(S)$, the coefficients are formal functions in $\bar{t}$ and polynomial functions in $q^{\gamma} e^{t^{2}}$ and $q^{\ell} e^{t^{1}}$ and the basic rational function $\mathbf{f}\left(q^{\ell} e^{t^{1}}\right)$, defined in (1.1).

We start with an overview of the general ideas involved in the proof. The Picard-Fuchs system generated by $\square_{\ell}$ and $\square_{\gamma}$ is a perturbation of the Picard-Fuchs (hypergeometric) system associated with the (toric) fiber by operators in base divisors. The fiberwise toric case is a GKZ system, which by the theorem of Gelfand-Kapranov-Zelevinsky is a holonomic system of rank $(r+1)(r+2)$, the dimension of cohomology space of a fiber. It is also known that the GKZ system admits a Gröbner basis reduction to the holonomic system.

We apply this result in the following manner: We will construct a $\mathscr{D}$-module with basis $\partial^{z e}$ for $\mathbf{e} \in \Lambda^{+}$. We apply operators $z \partial_{t^{1}}$ and $z \partial_{t^{2}}$ and first-order operators $z \partial_{i}$ to this selected basis. Notice that

$$
\begin{aligned}
& \square_{\ell}=\left(1-(-1)^{r+1} q^{\ell} e^{t^{1}}\right)\left(z \partial_{t^{1}}\right)^{r+1}+\cdots, \\
& \square_{\gamma}=\left(z \partial_{t^{2}}\right)^{r+2}+\cdots .
\end{aligned}
$$


The Gröbner basis reduction allows one to reduce the differentiation order in $z \partial_{t^{1}}$ and $z \partial_{t^{2}}$ to a smaller one. In the process higher-order differentiation in the $z \partial_{i}$ will be introduced. Using the $I$-lift, the differentiation in the base direction with order higher than 1 can be reduced to order 1 by introducing more terms with strictly larger effective classes in $\mathrm{NE}(S)$. A refinement of these observations will lead to a proof, which is presented below.

Remark 4.9. In fact, neither the Gröbner basis nor the GKZ theorem will be needed, due to the simple feature of the Picard-Fuchs system we have for split ordinary flops.

Proof. Consider first the case of simple $P^{r}$ flops $(S=\mathrm{pt})$. In this special case the Gröbner basis is already at hand. The naive quantization of the canonical cohomology basis gives

$$
\partial^{z(i, j)}:=\left(z \partial_{t^{1}}\right)^{i}\left(z \partial_{t^{2}}\right)^{j}, \quad 0 \leqslant i \leqslant r, 0 \leqslant j \leqslant r+1 .
$$

Further differentiation in the $t^{1}$-direction leads to

$$
z \partial_{t^{1}} \partial^{z(i, j)}=\partial^{z(i+1, j)}
$$

It is clear that we only need to deal with the boundary case $i=r$, when the right-hand side goes beyond the standard basis.

Case $(i, j)=(r, 0)$. The equation $\square_{\ell}=\left(z \partial_{t^{1}}\right)^{r+1}-q^{\ell} e^{t^{1}}\left(z \partial_{t^{2}}-z \partial_{t^{1}}\right)^{r+1} \equiv 0$ modulo $I$ leads to

$$
\left(z \partial_{t^{1}}\right)^{r+1} \equiv \frac{q^{\ell} e^{t^{1}}}{1-(-1)^{r+1} q^{\ell} e^{t^{1}}} \sum_{k=1}^{r+1} C_{k}^{r+1}\left(z \partial_{t^{2}}\right)^{k}\left(-z \partial_{t^{1}}\right)^{r+1-k},
$$

which solves the case.

Case $(i, j)=(r, j \geqslant 1)$. For $j \geqslant 1$, notice that $\square_{\gamma}=z \partial_{t^{2}}\left(z \partial_{t^{2}}-z \partial_{t^{1}}\right)^{r+1}-q^{\gamma} e^{t^{2}} \equiv 0$ modulo $I$. Hence

$$
\begin{aligned}
\left(z \partial_{t^{1}}\right)^{r+1}\left(z \partial_{t^{2}}\right)^{j} & =q^{\ell} e^{t^{1}}\left(z \partial_{t^{2}}\right)^{j}\left(z \partial_{t^{2}}-z \partial_{t^{1}}\right)^{r+1} \\
& \equiv q^{\ell} e^{t^{1}}\left(z \partial_{t^{2}}\right)^{j-1} q^{\gamma} e^{t^{2}}=q^{\ell} e^{t^{1}} q^{\gamma} e^{t^{2}}\left(z \partial_{t^{2}}+z\right)^{j-1} .
\end{aligned}
$$

This in particular solves the other cases with $1 \leqslant j \leqslant r+1$.

Similarly, differentiation in the $t^{2}$-direction gives

$$
z \partial_{t^{2}} \partial^{z(i, j)}=\partial^{z(i, j+1)}
$$

And we only need to deal with the boundary case $j=r+1$.

Case $(i, j)=(0, r+1) . \quad$ First of all, $\square_{\gamma} I=0$ leads to

$$
\begin{aligned}
\left(z \partial_{t^{2}}\right)^{r+2} & \equiv-(-1)^{r+1}\left(z \partial_{t^{1}}\right)^{r+1} z \partial_{t^{2}}-\sum_{k=1}^{r} C_{k}^{r+1}\left(z \partial_{t^{2}}\right)^{k+1}\left(-z \partial_{t^{1}}\right)^{r+1-k}+q^{\gamma} e^{t^{2}} \\
& =\left(1-(-1)^{r+1} q^{\ell} e^{t^{1}}\right) q^{\gamma} e^{t^{2}}-\sum_{k=1}^{r}(-1)^{r+1-k} C_{k}^{r+1} \partial^{z(r+1-k, k+1)},
\end{aligned}
$$

which solves the case. 


\section{INVARIANCE OF QUANTUM RINGS II}

Case $(i, j)=(i \geqslant 1, r+1)$. By further differentiating $t^{1}$ in (4.8) and in (4.7), we get

$$
\begin{aligned}
\left(z \partial_{t^{1}}\right)^{i}\left(z \partial_{t^{2}}\right)^{r+2} \equiv & \left(z \partial_{t^{1}}\right)^{i} q^{\gamma} e^{t^{2}}-(-1)^{r+1}\left(z \partial_{t^{1}}\right)^{i} q^{\ell} e^{t^{1}} q^{\gamma} e^{t^{2}} \\
& -\sum_{k=1}^{r}(-1)^{r+1-k} C_{k}^{r+1}\left(z \partial_{t^{1}}\right)^{r+1+(i-k)}\left(z \partial_{t^{2}}\right)^{k+1} \\
= & q^{\gamma} e^{t^{2}}\left(z \partial_{t^{1}}\right)^{i}-(-1)^{r+1} q^{\ell} e^{t^{1}} q^{\gamma} e^{t^{2}}\left(z \partial_{t^{1}}+z\right)^{i} \\
& -\sum_{k=i+1}^{r}(-1)^{r+1-k} C_{k}^{r+1} \partial^{z(r+i+1-k, k+1)} \\
& -q^{\ell} e^{t^{1}} q^{\gamma} e^{t^{2}} \sum_{k=1}^{i}(-1)^{r+1-k} C_{k}^{r+1}\left(z \partial_{t^{1}}+z\right)^{i-k}\left(z \partial_{t^{2}}+z\right)^{k} .
\end{aligned}
$$

This in particular solves the remaining cases with $1 \leqslant i \leqslant r$.

An important observation of the above calculation of the matrices $C_{1}(z, q)$ and $C_{2}(z, q)$ is that $C_{i}$ is constant in $z$ modulo $q^{\gamma}$. Moreover, $q^{d_{2} \gamma}$ appears only in the case $d_{2}=1$.

Now we consider the case with base $S$. The Picard-Fuchs equations are

$$
\begin{aligned}
& \square_{\ell}=\prod_{j=0}^{r} z \partial_{h+L_{j}}-q^{\ell} e^{t^{1}} \prod_{j=0}^{r} z \partial_{\xi-h+L_{j}^{\prime}}, \\
& \square_{\gamma}=z \partial_{\xi} \prod_{j=0}^{r} z \partial_{\xi-h+L_{j}^{\prime}}-q^{\gamma} e^{t^{2}} .
\end{aligned}
$$

Recall that for a basis element $T_{\mathbf{e}}=\bar{T}_{s} h^{i} \xi^{j}$ in its canonical presentation $(0 \leqslant i \leqslant r, 0 \leqslant j \leqslant r+1)$, we defined its naive quantization

$$
\hat{T}_{\mathbf{e}}=\partial^{z \mathbf{e}}=z \partial_{\bar{t}^{s}}\left(z \partial_{t^{1}}\right)^{i}\left(z \partial_{t^{2}}\right)^{j} .
$$

The above calculations (4.6)-(4.9) need to be corrected by adding more differential symbols which may consist of higher derivatives in base divisors $z \partial_{L_{j}}$ and $z \partial_{L_{j}^{\prime}}$ instead of a single $z \partial_{\bar{t}^{s}}$. Thus they are not yet in the desired form (4.11). The $I$-lift (4.4) helps to reduce higher derivatives in the base to first-order ones. Although new derivatives $D_{\bar{\beta}}$ may appear during this reduction, it is crucial to notice that they all come with non-trivial classes $q^{\bar{\beta}^{I}}$.

With these preparations, we will prove the theorem by constructing

$$
C_{a, \bar{\beta}}(z)=\sum_{\beta \mapsto \bar{\beta}} C_{a ; \beta}(z) q^{\beta}
$$

for any fixed $\bar{\beta} \in \mathrm{NE}(S)$.

For $\bar{\beta}=0$, the $I$-lift (4.4) introduces no further derivatives: $D_{\bar{\beta}=0}(z)=\mathrm{Id}$. Thus higherorder differentiations in the $\bar{t}^{s}$ can all be reduced to the first order. Notice that in (4.10) all the corrected terms have $\left(z \partial_{t^{1}}\right)^{i}\left(z \partial_{t^{2}}\right)^{j}$ in the canonical range, hence (4.6)-(4.9) plus (4.4) lead to the desired matrix $C_{a ; \bar{\beta}=0}(z)$.

Given $\bar{\beta} \in \mathrm{NE}(S)$, to determine the coefficient $C_{a, \bar{\beta}}$ from calculating $z \partial_{a}\left(\partial^{z \mathbf{e}}\right)$, it is enough to consider the restriction of (4.4) to the finite sum over $\bar{\beta}^{\prime} \leqslant \bar{\beta}$. We repeatedly apply the following two constructions:

(i) The double derivative in the base can be reduced to a single derivative by (4.4). If a new non-trivial derivative $D_{\bar{\beta}_{1}^{I}}(z)$ is introduced, then we get a new higher-order term with respect 


\section{Y.-P. LeE, H.-W. Lin ANd C.-L. WANG}

to $\mathrm{NE}(S)$ because the factor $q^{\bar{\beta}_{1}^{I}}$ is added; thus such processes will produce classes with image outside $\mathrm{NE}_{\leqslant \bar{\beta}}(S)$ in finitely many steps. In fact the only term in (4.4) not increasing the order in $\mathrm{NE}(S)$ is given by

$$
\bar{C}_{a j ; \bar{\beta}=0}^{k} z \partial_{k} .
$$

This is precisely the structural constant of the cup product on $H(S)$, which is non-zero only if

$$
\operatorname{deg} \bar{T}_{a}+\operatorname{deg} \bar{T}_{j}=\operatorname{deg} \bar{T}_{k} .
$$

Hence $\operatorname{deg} \bar{T}_{k} \geqslant \operatorname{deg} \bar{T}_{a}$, with equality only if $\bar{T}_{j}=1$, which may occur only for the first step. Any further reduction of double derivatives $z \partial_{k} z \partial_{l}$ in the base into a single derivative $z \partial_{m}$ must then increase the cohomology degree $\operatorname{deg} \bar{T}_{m}>\operatorname{deg} \bar{T}_{k}$ if the order in $\operatorname{NE}(S)$ is not increased. It is clear that the process stops in finitely many steps.

(ii) Each time we have terms not in the reduced form (4.11), we perform the Picard-Fuchs reduction (4.6)-(4.9) with correction terms. After the first step in simplifying $z \partial_{t^{1}}\left(\partial^{z \mathbf{e}}\right)$ and $z \partial_{t^{2}}\left(\partial^{z \mathbf{e}}\right)$, in all the remaining steps we face such a situation only when we have non-trivial terms $D_{\bar{\beta}_{1}^{I}}(z)$ from construction (i). As before this produces classes with image outside $\mathrm{NE}_{\leqslant \bar{\beta}}(S)$ in finitely many steps.

Combining constructions (i) and (ii), we obtain $C_{a ; \bar{\beta}}$ in finitely many steps. It is clearly polynomial in $z, q^{\gamma} e^{t^{2}}, q^{\ell} e^{t^{1}}$ and $\mathbf{f}\left(q^{\ell} e^{t^{1}}\right)$ since this holds for each step.

THEOREM 4.10 (Naturality). The system is $\mathscr{F}$-invariant. That is, $\mathscr{F} C_{a}(\hat{t}) \cong C_{a}^{\prime}(\mathscr{F} \hat{t})$.

Proof. We have seen the $\mathscr{F}$-invariance of the Picard-Fuchs systems. It remains to show the $\mathscr{F}$-invariance of the $I$-lift of the base Dubrovin connection, up to modification by $\square_{\ell}$ and $\square_{\gamma}$.

By (4.4), the simplest situation to achieve such an invariance is the case $\mathscr{F} \bar{\beta}^{I}=\bar{\beta}^{I^{\prime}}$, since then $\mathscr{F} D_{\bar{\beta}^{I}}(z)=D_{\bar{\beta}^{I^{\prime}}}^{\prime}(z)$ as well.

Indeed, when $\mu+\mu^{\prime} \geqslant 0$ for a curve class $\bar{\beta}$, we do have

$$
\begin{aligned}
\mathscr{F} \bar{\beta}^{I} & =\mathscr{F}\left(\bar{\beta}-\mu \ell-\left(\mu+\mu^{\prime}\right) \gamma\right)=\bar{\beta}+\mu \ell^{\prime}-\left(\mu+\mu^{\prime}\right)\left(\ell^{\prime}+\gamma^{\prime}\right) \\
& =\bar{\beta}-\mu^{\prime} \ell^{\prime}-\left(\mu+\mu^{\prime}\right) \gamma^{\prime}=\bar{\beta}^{I^{\prime}} .
\end{aligned}
$$

It remains to analyze the case $\mu+\mu^{\prime}<0$ for $\bar{\beta}$. In this case,

$$
\mathscr{F} \bar{\beta}^{I}-\bar{\beta}^{I^{\prime}}=\bar{\beta}+\mu \ell^{\prime}-\left(\bar{\beta}-\mu^{\prime} \ell^{\prime}\right)=\left(\mu+\mu^{\prime}\right) \ell^{\prime}=-\delta \ell^{\prime},
$$

where $\delta:=-\left(\mu+\mu^{\prime}\right)>0$ is the finite gap. Thus

$$
\mathscr{F} q^{\bar{\beta}^{I}-\delta \ell}=q^{\bar{\beta}^{I^{\prime}}}
$$

and this suggests that we should try to decrease $\bar{\beta}^{I}$ by $\ell$ a total of $\delta$ times.

In other words, we should expect to have another valid lift:

$$
z \partial_{i} z \partial_{j} I=\sum_{k, \bar{\beta}} q^{\bar{\beta}^{I}-\delta \ell} e^{D \cdot\left(\bar{\beta}^{I}-\delta \ell\right)} \bar{C}_{i j, \bar{\beta}}^{k}(\bar{t}) z \partial_{k} D_{\bar{\beta}^{I}-\delta \ell}(z) I .
$$

This is easy to check: Notice that $n_{i}\left(\bar{\beta}^{I}-\delta \ell\right)=n_{i}\left(\bar{\beta}^{I}\right)+\delta>0$ and $n_{i}^{\prime}\left(\bar{\beta}^{I}-\delta\right)=n_{i}^{\prime}\left(\bar{\beta}^{I}\right)-\delta$, which is also $n_{i}^{\prime}\left(\bar{\beta}+\mu^{\prime} \ell\right)=\mu^{\prime}-\mu_{i}^{\prime} \geqslant 0$ (cf. the gap in (4.2)). The value $n_{r+1}^{\prime} \geqslant 0$ is unchanged. Thus, the operator $D_{\bar{\beta}^{I}-\delta \ell}$ is well defined, though $\bar{\beta}^{I}-\delta \ell$ may not be effective. By Theorem 4.6, we see that (4.12) is also a lift and the theorem is proved. 


\section{INVARIANCE OF QUANTUM RINGS II}

\subsection{Reduction to the canonical form: the final proof}

We will construct a gauge transformation $B$ to eliminate all $z$-dependence of $C_{a}$. The final system is then equivalent to the Dubrovin connection on $\mathrm{QH}(X)$. Such a procedure is well known in the small quantum cohomology of Fano-type examples or in the context of abstract quantum cohomology. (See, for example, [Gue08] and references therein.) Here we will also need to take into account the role played by the generalized mirror transformation (GMT) $\tau(\hat{t})$.

In fact, $B$ is nothing more than the Birkhoff factorization introduced before:

$$
\left(\partial^{z \mathbf{e}} I(\hat{t})\right)=(z \nabla J)(\tau) B(\tau),
$$

valid at the generalized mirror point $\tau=\tau(\hat{t})$. Thus $B$ exists uniquely via an inductive procedure. However, the analytic (formal) dependence of $B$ is not manifest if one proceeds in this direction, as the procedure involves $J$ and $I$, and the analytic dependence holds for neither. Therefore, it is not clear how to prove $\mathscr{F} B \cong B^{\prime}$ up to analytic continuation.

In this subsection we will proceed in a slightly different, but ultimately equivalent, way. Namely, we study instead the gauge transformation $B$ directly from the differential system

$$
z \partial_{a}\left(\partial^{z \mathbf{e}} I\right)=\left(\partial^{z \mathbf{e}} I\right) C_{a} .
$$

Even though the solutions $I$ are not $\mathscr{F}$-invariant, the system is $\mathscr{F}$-invariant by Theorem 4.10. This system can be analyzed inductively with respect to the partial ordering of the Mori cone on the base $\mathrm{NE}(S)$.

Substituting (4.13) into (4.14), we get $z \partial_{a}(\nabla J) B+z(\nabla J) \partial_{a} B=(\nabla J) B C_{a}$, hence

$$
z \partial_{a}(\nabla J)=(\nabla J)\left(-z \partial_{a} B+B C_{a}\right) B^{-1}=:(\nabla J) \tilde{C}_{a} .
$$

We note the subtlety in the meaning of $\tilde{C}_{a}(\hat{t})$. Let $\tau=\sum \tau^{\mu} T_{\mu}$. Then the QDE reads as

$$
z \partial_{\mu}(\nabla J)(\tau)=(\nabla J)(\tau) \tilde{C}_{\mu}(\tau),
$$

where $\tilde{C}_{\mu}(\tau)$ is the structure matrix of quantum multiplication at the point $\tau \in H(X)$. Then

$$
z \partial_{a}(\nabla J)=\sum_{\mu} \frac{\partial \tau^{\mu}}{\partial t^{a}} z \partial_{\mu}(\nabla J)=(\nabla J) \sum_{\mu} \tilde{C}_{\mu} \frac{\partial \tau^{\mu}}{\partial t^{a}}
$$

hence

$$
\tilde{C}_{a}(\hat{t}) \equiv \sum_{\mu} \tilde{C}_{\mu}(\tau(\hat{t})) \frac{\partial \tau^{\mu}}{\partial t^{a}}(\hat{t}) .
$$

In particular, $\tilde{C}_{a}$ is independent of $z$.

Taking this into account, (4.15) in fact is equivalent to

$$
\tilde{C}_{a}=B_{0} C_{a ; 0} B_{0}^{-1}
$$

$\left(B_{0}^{-1}:=\left(B^{-1}\right)_{0}\right)$ and the cancellation equation

$$
z \partial_{a} B=B C_{a}-B_{0} C_{a ; 0} B_{0}^{-1} B,
$$

where the subscript 0 stands for the coefficients of $z^{0}$ in the $z$-expansion.

Our plan is to analyze $B=B(z)$ with respect to the weight $w:=\left(\bar{\beta}, d_{2}\right) \in W$, which carries a natural partial ordering. The initial condition is $B_{w=(0,0)}=\mathrm{Id}$, since we have seen that for $w=(0,0)$, the only $z$-constant term of $C_{a}$ is $C_{a ;(0,0), 0} z^{0}$. The total $z$-constant terms in $(4.18)$ are trivially compatible. They are $-B_{0} C_{a ; 0}$ on both sides. 
Now, we perform the induction on $W$. Suppose that $B_{w^{\prime}}$ satisfies $\mathscr{F} B_{w^{\prime}}=B_{w^{\prime}}^{\prime}$ for all $w^{\prime}<w$. Then

$$
z \partial_{a} B_{w}=\sum_{w_{1}+w_{2}=w} B_{w_{1}} C_{a ; w_{2}}-\sum_{w_{1}+w_{2}+w_{3}+w_{4}=w} B_{w_{1}, 0} C_{a ; w_{2}, 0} B_{w_{3}, 0}^{-1} B_{w_{4}}
$$

Write $C_{a ; w}=\sum_{j=0}^{m(w)} C_{a ; w, j} z^{j}$ and $B_{w}=\sum_{j=0}^{n(w)} B_{w, j} z^{j}$. Then (4.19) implies

$$
n(w)=\max _{w^{\prime}<w}\left(n\left(w^{\prime}\right)+m\left(w-w^{\prime}\right)\right)-1 .
$$

Notice that on the right-hand side all $B$-terms have degree strictly smaller than $w$ except

$$
B_{w} C_{a ;(0,0)}-C_{a ;(0,0)} B_{w}+B_{w, 0} C_{a ;(0,0)}-C_{a ;(0,0)} B_{w, 0}^{-1},
$$

which has maximal $z$-degree at most $n(w)$. Moreover, by descending induction on the $z$-degree, to determine $B_{w, j}$ we need only $B_{w^{\prime}}$ with $w^{\prime}<w$ or $B_{w, j^{\prime}}$ with $j^{\prime}>j$, which are all $\mathscr{F}$-invariant by induction. Hence the difference satisfies

$$
\partial_{a}\left(\mathscr{F} B_{w, j}-B_{w, j}^{\prime}\right)=0 .
$$

The functions involved are all formal in $\bar{t}$ and analytic in $t^{1}$ and $t^{2}$, and without constant term $\left(B_{w=(0,0)}=\mathrm{Id}\right)$. Hence $\mathscr{F} B_{w, j}=B_{w, j}^{\prime}$.

To summarize, we have proved that for any $\hat{t}=\bar{t}+D \in H(S) \oplus \mathbb{C} h \oplus \mathbb{C} \xi$,

$$
\mathscr{F} B(\tau(\hat{t})) \cong B^{\prime}\left(\tau^{\prime}(\hat{t})\right) \text {. }
$$

In particular, by (4.17) this implies the $\mathscr{F}$-invariance of $\tilde{C}_{a}(\hat{t})$. In more explicit terms, we have the $\mathscr{F}$-invariance of

$$
\tilde{C}_{a \nu}^{\kappa}=\sum_{n \geqslant 0, \mu} \frac{q^{\beta}}{n !} \frac{\partial \tau^{\mu}(\hat{t})}{\partial t^{a}}\left\langle T_{\mu}, T_{\nu}, T^{\kappa}, \tau(\hat{t})^{n}\right\rangle_{\beta}
$$

for arbitrary (basis elements) $T_{\nu}, T^{\kappa} \in H(X)$.

The very special case $T_{\nu}=1$ leads to non-trivial invariants only for the 3 -point classical invariant $(n=0)$ and $\beta=0$, and also $\mu=\kappa$. Since $\kappa$ is arbitrary, we have thus proved the $\mathscr{F}$-invariance of $\partial_{a} \tau$. Then

$$
\partial_{a}\left(\mathscr{F} \tau-\tau^{\prime}\right)=\mathscr{F} \partial_{a} \tau-\partial_{a} \tau^{\prime}=0 .
$$

Again, since $\tau(\hat{t})=\hat{t}$ for $\left(\bar{\beta}, d_{2}\right)=(0,0)$, this proves

$$
\mathscr{F} \tau=\tau^{\prime} .
$$

Remark 4.11. The matrix $\tilde{C}_{a}$ is the derivative of the 2-point (Green) function at $\tau(\hat{t})$,

$$
\tilde{C}_{a \nu}^{\kappa}=\frac{\partial}{\partial t^{a}}\left\langle\left\langle T_{\nu}, T^{\kappa}\right\rangle\right\rangle(\tau) .
$$

Now, we may finish the proof of the quantum invariance (Theorem 1.3).

Proof. Since we have established the analytic continuation of $B$ (hence $P$ ) and $\tau$, by Proposition 2.11 (reduction to special BF/GMT with $\xi$-class) and Lemma 3.12 (naive quasi-linearity with $\xi$-class) the invariance of the quantum ring is proved.

Remark 4.12. We sketch an alternative shortcut to the proof to minimize the usage of extremal functions and completely get rid of the quasi-linearity reduction.

Indeed, by degeneration reduction (Section 4 of Part I [LLW16]), the quantum invariance problem is reduced to local models for descendent invariants of special type. Theorem 5.2 of 


\section{INVARIANCE OF QUANTUM RINGS II}

[LLW16] then eliminates the necessity of using $\psi$-classes and we only need to prove the invariance of the quantum ring for local models.

Now for split flops, the Birkhoff factorization matrix $B(z)$ exists uniquely. Then the quantum Leray-Hirsch theorem (Theorem 4.8) produces the matrix $C_{a}(z)$ which satisfies the analytic continuation property. The analytic continuation of $B(z)$ is then deduced from it. In particular, (4.17) gives the analytic continuation of $\tilde{C}_{a}(\hat{t})$, namely (4.20), and then of $\tau(\hat{t})$.

Now we apply the reduction method used in the proof of Proposition 2.11, with the role of special insertion $\tau_{k} a \xi$ replaced by three primary insertions $T_{a}, T_{\nu}, T^{\kappa}$ with $T_{a} \in H(S)$ and $T_{\nu}$, $T^{\kappa} \in H(X)$ arbitrary. We can do so because $\partial \tau / \partial t^{a}=T_{a}+\cdots$. Notice that since $n \geqslant 3$, the divisor reconstructions we need can all be performed within primary invariants.

Namely, using [LLW16, equation (3.1)] for $h$ and $\xi$, we may reconstruct any $n$-point primary invariants with $n \geqslant 3$ from the primary invariant with only two general insertions not from $H(S)$. As in step 2 of the proof of [LLW16, Theorem 5.4], moving the $\xi$-class will always be $\mathscr{F}$-compatible, while moving the $h$-class to an insertion $t_{i} h^{r}$ may generate a topological defect. The key point is that this defect can be cancelled out by the extremal quantum corrections from some diagonal splitting term. (In fact, this is the building block of our determination of the extremal invariants in Section 3 of Part I [LLW16].)

This leads to a logically shorter and more conceptual proof of the quantum invariance theorem.

We present the complete argument for at least two reasons. First, the quantum correction part (extremal case) works for non-split flops as well. Second, the BF/GMT algorithm, together with the divisorial reconstruction, provides an effective method to determine all genus zero descendent (not just primary) invariants for any split toric bundle.

\section{Examples of the quantum Leray-Hirsch theorem}

\subsection{The toy example}

We consider the Hirzebruch surface $X=\Sigma_{-1}$ which is the $P^{1}$ bundle over $P^{1}$ associated with the vector bundle $\mathscr{O} \oplus \mathscr{O}(1)$. The GW theory on $X$ can be easily determined by the classical method. However, we will apply the quantum Leray-Hirsch theorem to it and compare the result with that obtained by the classical method.

Write $H(S)=H\left(P^{1}\right)=\mathbb{C}[p] /\left(p^{2}\right)$. By the Leray-Hirsch theorem, $H(X)=H(S)[h] /\langle h(h+p)\rangle$ has rank $N=4$. Consider the basis $\left\{T_{i} \mid 1 \leqslant i \leqslant 4\right\}$ given in the following order:

$$
1, h, p, h p \text {. }
$$

The dual basis $\left\{T^{i}\right\}$ is easily seen to be given by

$$
h p, p, h+p, 1 .
$$

We write $q=q^{\ell} e^{t}$ and $\bar{q}=q^{b} e^{\bar{t}}$, where $b=[S] \cong\left[P^{1}\right]$. The Picard-Fuchs operator is

$$
\square_{\ell}=\left(z \partial_{h}\right)\left(z \partial_{h+p}\right)-q \text {. }
$$

It leads to

$$
\left(z \partial_{h}\right)^{2}=q-\left(z \partial_{h}\right)\left(z \partial_{p}\right) .
$$

Since $H(S)=H^{0}(S) \oplus H^{2}(S)=\mathbb{C} 1 \oplus \mathbb{C} p$ consists of small parameters only, the small and 
big quantum rings coincide. It is easy to compute its QDE:

$$
z \partial_{p}\left(z \partial_{1}, z \partial_{p}\right)=\left(z \partial_{1}, z \partial_{p}\right)\left(\begin{array}{cc}
0 & \bar{q} \\
1 & 0
\end{array}\right)
$$

Since $b^{I}=b-\ell$, we get $D_{b^{I}}(z)=z \partial_{h}$. We find the lift of the QDE to be

$$
\left(z \partial_{p}\right)^{2}=\bar{q} q^{-1} z \partial_{h} .
$$

Using (5.1) and (5.2), we calculate the matrix $C_{t^{a}}$ of the action of $z \partial_{t^{a}}=z \partial_{h}$ or $z \partial_{p}$ on $\hat{T}_{i}$ as $z \partial_{t^{a}} \hat{T}_{j}=\sum_{k} C_{t^{a} j}^{k}(z) \hat{T}_{k}$ modulo $I^{X}$. Then

$$
C_{h}=\left[\begin{array}{cccc}
0 & q & 0 & -\bar{q} \\
1 & 0 & 0 & z \bar{q} q^{-1} \\
0 & 0 & 0 & q \\
0 & -1 & 1 & \bar{q} q^{-1}
\end{array}\right], \quad C_{p}=\left[\begin{array}{cccc}
0 & 0 & 0 & \bar{q} \\
0 & 0 & \bar{q} q^{-1} & -z \bar{q} q^{-1} \\
1 & 0 & 0 & 0 \\
0 & 1 & 0 & -\bar{q} q^{-1}
\end{array}\right]
$$

Here the indices $k$ and $j$ correspond to the row and column indices, respectively.

We solve $B$ from $C_{h}$ and $C_{p}$ by the recursive equation (4.19): $B_{2,4}=-\bar{q} q^{-1}$,

$$
B=\left[\begin{array}{cccc}
1 & 0 & 0 & 0 \\
0 & 1 & 0 & -\bar{q} q^{-1} \\
0 & 0 & 1 & 0 \\
0 & 0 & 0 & 1
\end{array}\right]
$$

Looking at the first column vector, it implies that in $J=P I$, one needs no Birkhoff factorization $(P(z)=1)$ and the mirror transformation reduces to the identity $\tau(\hat{t})=\hat{t}$. The full matrix system requires a basis in all directions which uses the full matrix $B$, and non-trivial Birkhoff factorization is required:

$$
B=I_{4}-\bar{q} q^{-1} e_{2,4}, \quad B^{-1}=I_{4}+\bar{q} q^{-1} e_{2,4} .
$$

Using this we get $\tilde{C}_{t^{a}}$ from (4.17): $\tilde{C}_{t^{a}}=B_{0} C_{t^{a} ; 0} B_{0}^{-1}$, which is a minor adjustment of the matrix $C_{t^{a}}$. We have

$$
\tilde{C}_{h}=\left[\begin{array}{cccc}
0 & q & 0 & 0 \\
1 & \bar{q} q^{-1} & -\bar{q} q^{-1} & 0 \\
0 & 0 & 0 & q \\
0 & -1 & 1 & 0
\end{array}\right], \quad \tilde{C}_{p}=\left[\begin{array}{cccc}
0 & 0 & 0 & \bar{q} \\
0 & -\bar{q} q^{-1} & \bar{q} q^{-1} & 0 \\
1 & 0 & 0 & 0 \\
0 & 1 & 0 & 0
\end{array}\right]
$$

By setting $\hat{t}=0$, we get $q=q^{\ell}$ and $\bar{q}=q^{b}$. Thus we can read out the corresponding 3-point invariants from the tables above. For example, we look at the entries at $(2,3)$ :

$$
\begin{aligned}
& \tilde{C}_{h 3}^{2}=\left\langle T_{2}, T_{3}, T^{2}\right\rangle=\langle h, p, p\rangle=-q^{-\ell} q^{b}, \\
& \tilde{C}_{p 3}^{2}=\left\langle T_{3}, T_{3}, T^{2}\right\rangle=\langle p, p, p\rangle=q^{-\ell} q^{b} .
\end{aligned}
$$

By the classical method, we can write down the $I$-function: for $\beta=d \ell+s b$,

$$
I_{\beta}^{X}=\frac{q^{d \ell} q^{s b}}{\prod_{1}^{s}(p+m z)^{2} \prod_{1}^{d}(h+m z) \prod_{1}^{d+s}(h+p+m z)}=O\left(z^{-2}\right)
$$

This implies $J^{X}=I^{X}$. Also, we find that $I_{\beta}^{X}=O\left(z^{-3}\right)$ except when $s=1$ and $d=-1$, and in that case the coefficient of $z^{-2}$ is $h$. It tells us that $\langle p\rangle=q^{-\ell} q^{b}$ and $\langle h\rangle=-q^{-\ell} q^{b}$. 


\section{INVARIANCE OF QUANTUM RINGS II}

(Here we have used $h^{2}=-h p$.) By the divisor axiom, $\langle h, p, p\rangle=\delta_{h} \delta_{p}\langle p\rangle=-q^{-\ell} q^{b}$. Similarly, $\langle p, p, p\rangle=\delta_{p} \delta_{p}\langle p\rangle=q^{-\ell} q^{b}$. These results coincide with (5.3).

Remark 5.1. Notice that we state and prove the quantum Leray-Hirsch theorem (Theorem 4.8) for certain double projective bundles (of split type) in order to apply it to the analytic continuation problem under flops. The same proof shows that it holds true for projective bundles, and more generally for iterated projective bundles (of split type).

\subsection{An example with non-trivial BF/GMT}

Consider the $P^{1}$ flop $f: X \rightarrow X^{\prime}$ with bundle data

$$
\left(S, F, F^{\prime}\right)=\left(P^{1}, \mathscr{O} \oplus \mathscr{O}, \mathscr{O} \oplus \mathscr{O}(1)\right)
$$

Write $H(S)=\mathbb{C}[p] /\left(p^{2}\right)$ with Chern polynomials

$$
f_{F}(h):=h^{2}, \quad f_{N \oplus \mathscr{O}}(\xi):=\xi(\xi-h)(\xi-h+p) .
$$

Then $H=H(X)=H(S)[h, \xi] /\left(f_{F}, f_{N \oplus \mathscr{O}}\right)$ has dimension $N=12$ with basis $\left\{T_{i} \mid 0 \leqslant i \leqslant 11\right\}$ equal to

$$
1, h, \xi, p, h \xi, h p, \xi^{2}, \xi p, h \xi^{2}, h \xi p, \xi^{2} p, h \xi^{2} p
$$

Set $q_{1}=q^{\ell} e^{t^{1}}, q_{2}=q^{\gamma} e^{t^{2}}, \bar{q}=q^{b} e^{t^{3}}$, where $b=[S] \cong\left[P^{1}\right]$, and $\mathbf{f}=\mathbf{f}\left(q_{1}\right)$. The Picard-Fuchs operators are

$$
\begin{aligned}
& \square_{\ell}=\left(z \partial_{h}\right)^{2}-q_{1} z \partial_{\xi-h} z \partial_{\xi-h+p}, \\
& \square_{\gamma}=z \partial_{\xi} z \partial_{\xi-h} z \partial_{\xi-h+p}-q_{2} .
\end{aligned}
$$

They lead to

$$
\begin{aligned}
\left(z \partial_{h}\right)^{2} & =\mathbf{f}\left(z \partial_{\xi}\right)^{2}-\mathbf{f} z \partial_{p} z \partial_{h}+\mathbf{f} z \partial_{p} z \partial_{\xi}-2 \mathbf{f} z \partial_{h} z \partial_{\xi} \\
\left(z \partial_{\xi}\right)^{3} & =q_{2}\left(1-q_{1}\right)-z \partial_{p}\left(z \partial_{\xi}\right)^{2}+2 z \partial_{h}\left(z \partial_{\xi}\right)^{2}+z \partial_{p} z \partial_{h} z \partial_{\xi}
\end{aligned}
$$

As before, $H(S)=H^{0}(S) \oplus H^{2}(S)=\mathbb{C} 1 \oplus \mathbb{C} p$ has only small parameters and QDE given by

$$
z \partial_{p}\left(z \partial_{1}, z \partial_{p}\right)=\left(z \partial_{1}, z \partial_{p}\right)\left(\begin{array}{cc}
0 & \bar{q} \\
1 & 0
\end{array}\right)
$$

The real difference from the previous $((0,0),(0,-1))$ case starts with the lift of this QDE. Now $b^{I}=b-\gamma$, so we get $D_{b}=z \partial_{\xi} z \partial_{\xi-h}$, and the lift becomes

$$
\left(z \partial_{p}\right)^{2}=\bar{q} q_{2}^{-1} z \partial_{\xi} z \partial_{\xi-h} .
$$

By (5.4)-(5.6), following the steps in the proof of Theorem 4.8, we calculate $C_{a}$ in $z \partial_{a} \hat{T}_{j}=$ $\sum_{k} C_{a j}^{k}(z) \hat{T}_{k}$ modulo $I^{X}$. This is a lengthy yet straightforward calculation. For simplicity let 
Y.-P. LeE, H.-W. Lin And C.-L. WANG

$q^{*}=\bar{q} q_{2}^{-1}$ be the chosen admissible lift and set $\mathbf{g}=\mathbf{f}\left(q^{*}\right), A=q_{2}-q_{1} q_{2}, S=q_{2}+q_{1} q_{2}$. We get

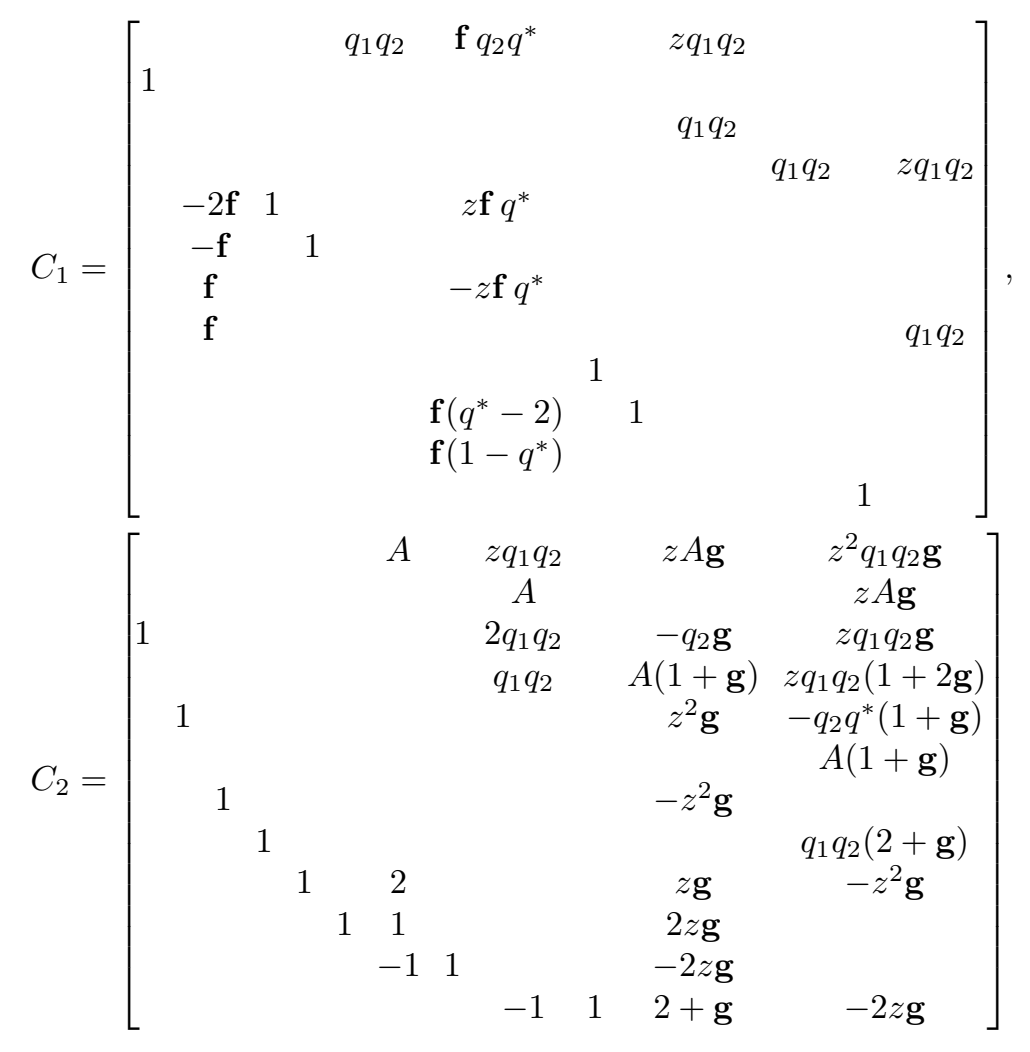

and

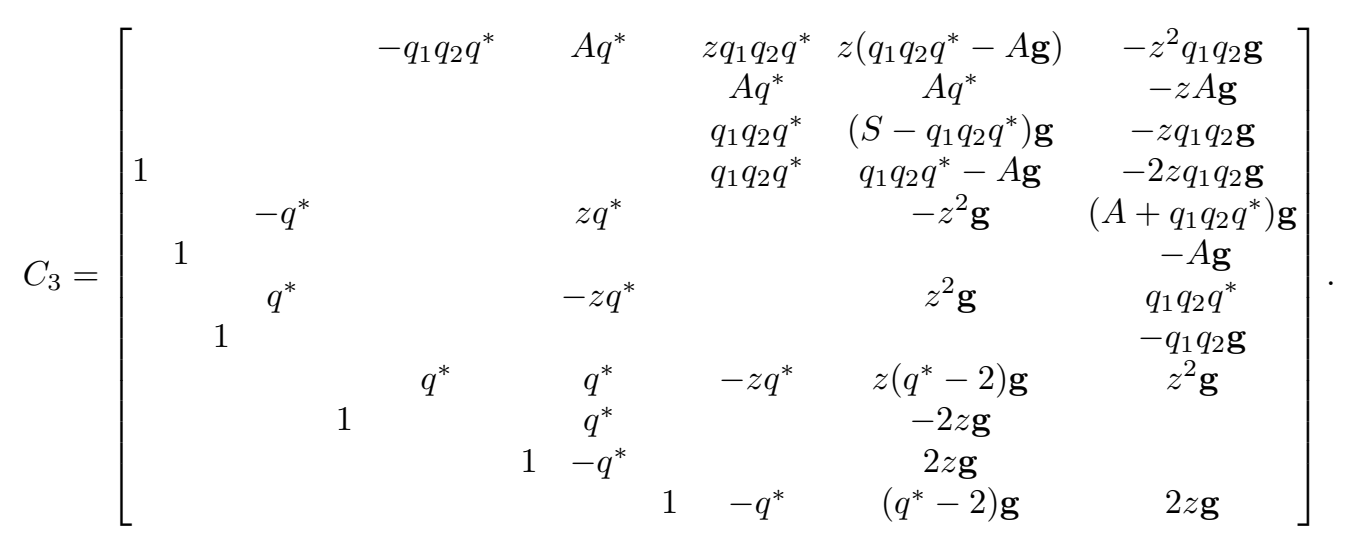

The appearance of $\mathbf{f}$ and $\mathbf{g}$ demonstrates the analytic dependence on the parameters and explains the validity of analytic continuation. It is now possible to solve the gauge transform $B$ inductively on $w=\left(\bar{\beta}, d_{2}\right)$. The formulae are complicated and the details are thus omitted.

Remark 5.2. These examples were contained in the manuscript prepared by the second and third authors and circulated at the MSJ Autumn meeting 2012, at Kyushu University.

\section{Appendix A. BF/GMT and regularization}

We consider a local split $P^{r}$ flop $f: X \rightarrow X^{\prime}$ over a general base $S$ and perform the BF/GMT algorithm from Section 2 simultaneously on $X$ and $X^{\prime}$. A mysterious cancellation arisen from the Birkhoff factorization, which is called regularization here, leads to the first step of analytic 


\section{INVARIANCE OF QUANTUM RINGS II}

continuation by transforming a rational function into its polynomial part in a canonical fashion. (See Proposition A.6.)

This result might lead one to believe that it is possible to prove the main results of this paper without the quantum Leray-Hirsch theorem. However, a closer look at the proof reveals the increasing complexity of the combinatorics and shows the limitation of this approach beyond the first step. In fact, the quantum Leray-Hirsch theorem implicitly implies the existence of all higher-order regularizations. A direct proof along the line presented here seems, however, a rather non-trivial combinatorial task.

\section{A.1 The fundamental rational functions $Q$ and $W_{\beta_{S}, d_{2}}$}

We start by recalling some of the basic set-up from Section 3 .

Consider a local split $P^{r}$ flop $f: X \rightarrow X^{\prime}$ with structure data $\left(S, F, F^{\prime}\right)$, where $F=\bigoplus_{i=0}^{r} L_{i}$ and $F^{\prime}=\bigoplus_{i=0}^{r} L_{i}^{\prime}$ are sums of line bundles. Let $a_{i}=c_{1}\left(L_{i}\right)+h$ and $b_{i}=c_{1}\left(L_{i}^{\prime}\right)+\xi-h$. For $\beta=\beta_{S}+d \ell+d_{2} \gamma$, set $\mu_{i}:=L_{i} \cdot \beta_{S}$ and $\mu_{i}^{\prime}:=L_{i}^{\prime} \cdot \beta_{S}$. Thus $a_{i} \cdot \beta=d+\mu_{i}$ and $b_{i} \cdot \beta=d_{2}-d+\mu_{i}^{\prime}$. Also recall that $\mu^{I}=\max _{i} \mu_{i}, \mu^{\prime I}=\max _{i} \mu_{i}^{\prime}$, and $\nu^{I}=\max \left\{\mu^{I}+\mu^{\prime I}, 0\right\}$. Let

$$
\lambda_{\beta}:=c_{1}(X / S) \cdot \beta=\left(c_{1}+c_{1}^{\prime}\right) \cdot \beta_{S}+(r+2) d_{2}=\sum\left(\mu_{i}+\mu_{i}^{\prime}\right)+(r+2) d_{2},
$$

which depends only on $\left(\beta_{S}, d_{2}\right)$. Then the hypergeometric modification takes on the form

$$
I=I\left(t^{1}, t^{2}, \bar{t}, z, z^{-1}\right)=e^{\left(t^{1} h+t^{2} \xi\right) / z} \sum_{\beta \in \operatorname{NE}(X)} q^{\beta} e^{d t^{1}+d_{2} t^{2}} I_{\beta}^{X / S} J_{\beta_{S}}^{S}(\bar{t})
$$

with relative factor

$$
I_{\beta}^{X / S}=z^{-\lambda_{\beta}} \frac{\Gamma(1+\xi / z)}{\Gamma\left(1+\xi / z+d_{2}\right)} \prod_{i=0}^{r} \frac{\Gamma\left(1+a_{i} / z\right)}{\Gamma\left(1+a_{i} / z+d+\mu_{i}\right)} \frac{\Gamma\left(1+b_{i} / z\right)}{\Gamma\left(1+b_{i} / z+d_{2}-d+\mu_{i}^{\prime}\right)} .
$$

The case $d_{2}<0$ leads to a $\xi$-factor, and then $\mathscr{F} I_{d_{2}}=I_{d_{2}}^{\prime}$, which contains only the $\mathscr{F} I$ effective range (by Lemma 3.12). In particular, the BF and GMT are all $\mathscr{F}$-compatible. So let $d_{2} \geqslant 0$. In this case, it is clear that the factor $\Gamma(1+\xi / z) / \Gamma\left(1+\xi / z+d_{2}\right)$ contains $\xi$ except for the $\xi$-constant term $1 /\left(d_{2}\right.$ !). Thus this factor needs no treatment and will be ignored in the following discussion. In other words, $I_{\beta}^{X / S}$ will be used as if this factor is 1 . For the same reason (the appearance of a $\xi$-factor), the $\mathrm{BF}$ is needed only if $\lambda_{\beta} \leqslant 0$.

Recall the rule for the directed product: for any $n \in \mathbb{Z}$,

$$
\frac{\Gamma(1+A)}{\Gamma(1+A+n+x)}=\frac{\Gamma(1+A)}{\Gamma(1+A+x)} \prod_{j=1}^{n}(A+j+x)^{-1} .
$$

Definition A.1. Given $\left(\beta_{S}, d_{2}\right)$, with $d_{2}>-\nu^{I}$, the fundamental rational function $Q(\vec{x})$ in $\vec{x}=\left(x_{0}, \ldots, x_{r}, y_{0}, \ldots, y_{r}\right)$ with cohomology-valued coefficients is defined by

$$
Q(\vec{x})=Q_{\beta_{S}, d_{2}}(\vec{x}):=\prod_{i=0}^{r} \prod_{j=1}^{\mu_{i}}\left(\frac{a_{i}}{z}+j+x_{i}\right)^{-1} \prod_{j=1}^{d_{2}+\mu_{i}^{\prime}}\left(\frac{b_{i}}{z}+j-y_{i}\right)^{-1} .
$$

Its 1-variable (diagonal) version $Q(x)$ is given by setting $x_{i}=x=y_{i}$ for all $i$. Abusing the notation, we write $\vec{x}=x$ for this specialization. 
In terms of $Q$, assuming (A.2), the product in $I_{\beta}^{X / S}$ is then the specialization of

$$
Q(\vec{x}) \prod_{i=0}^{r} \frac{\Gamma\left(1+a_{i} / z\right)}{\Gamma\left(1+a_{i} / z+x_{i}\right)} \frac{\Gamma\left(1+b_{i} / z\right)}{\Gamma\left(1+b_{i} / z-y_{i}\right)}=: Q(\vec{x}) I_{\vec{x} \ell}
$$

at $\vec{x}=d$. However, cancellations have to be carried out on the right-hand side of (A.3) for certain $\vec{x}=d \in \mathbb{Z}$ : When $x=d \in \mathbb{N}$, it is clear that $I_{d \ell}$ contains the factor

$$
\frac{\Theta_{r+1}}{z^{r+1}}:=\prod_{i=0}^{r} \frac{b_{i}}{z}
$$

However, for those $i$ with $d_{2}-d+\mu_{i}^{\prime} \geqslant 0$ (which exist when $\beta$ is $\mathscr{F} I$-effective), it is understood that the factor $b_{i} / z$ cancels out with the same term in the denominator of $Q(d)$. To make sense of the cancellation of $b_{i}$, we may temporarily treat the classes $a_{i}$ and $b_{i}$ as formal variables.

For those $i$ with $d+\mu_{i}<0$, the factor $a_{i} / z$ appears in the numerator. This is not the case for at least one $i$ (since $\beta$ is effective; otherwise the factor $\prod_{i=0}^{r} a_{i}=0$ appears). Thus the leading terms take on the form

$$
c(d) \prod_{d+\mu_{i}<0} \frac{a_{i}}{z} \prod_{d_{2}-d+\mu_{i}^{\prime}<0} \frac{b_{i}}{z}+\cdots
$$

in their $1 / z$-expansion. The leading expression changes as $d$ varies over the integer values. This motivates the following

Definition A.2. Given $\left(\beta_{S}, d_{2}\right)$, the class $\beta=\beta_{S}+d \ell+d_{2} \gamma \in \mathrm{NE}(X)$, as well as $d$, is said to be in the unstable range if $\beta$ is $\mathscr{F} I$-effective $\left(d \leqslant d_{2}+\mu^{\prime I}\right)$. Otherwise it is in the stable range $\left(d>d_{2}+\mu^{\prime I}\right)$.

In view of (A.3) and (A.4), the leading $z$-order of $I_{\beta}^{X / S}$ which admits infinite series in $d$ is at $z^{-\lambda_{\beta}-(r+1)}$. Any $z^{k}$ with $k>-\lambda_{\beta}-(r+1)$ supports only a finite number of $d$ and all of them are within the unstable range. For this reason, we consider the shifted expression

$$
W[r+1]\left(\vec{x}, z, z^{-1}\right):=z^{r+1} Q(\vec{x}) I_{\vec{x} \ell}
$$

to locate the first infinite series at the $z^{0}$-level (constant level).

Viewing $1 / z$ as $\Delta x_{i}=\Delta y_{i}$, the expression $W[r+1]$ is the multivariate extension in the multidirections $a_{i}$ and $-b_{i}$ of the similar expression $W(\vec{x})$ defined by setting $1 / z=0$ in $W[r+1]$ :

$$
W(\vec{x}):=\left.z^{r+1}\left(Q(\vec{x}) I_{\vec{x} \ell}\right)\right|_{1 / z=0} .
$$

Notice that $W(x)$ has poles at some $x=d$ if and only if a non-trivial positive $z$-power survives in $W[r+1](d)$. By our construction, $d$ must lie in the unstable range.

Remark A.3. This extension is unique under the normalization that $I_{\vec{x} \ell}=1$ at $\vec{x}=0$. Indeed, $I_{x \ell}\left(z^{-1}=0\right)=1 / \prod_{i=0}^{r} \Gamma(1+x) \Gamma(1-x)=(\sin \pi x / \pi x)^{r+1}$. The naive extension gives only $1 / \prod_{i=0}^{r} \Gamma\left(1+a_{i} / z+x\right) \Gamma\left(1+b_{i} / z-x\right)$. The extra factor $\prod_{i=0}^{r} \Gamma\left(1+a_{i} / z\right) \Gamma\left(1+b_{i} / z\right)$ is needed to recover $I_{x \ell}$.

For $x=d \in \mathbb{N}$, applying the Taylor series for $\log (1 \pm t)$ to each $a_{i}$ or $b_{i}$ separately and then 
taking a product, we get

$$
\begin{aligned}
I_{d \ell} & =\prod_{i=0}^{r} \prod_{j=-d+1}^{0}\left(b_{i} / z+j\right) \prod_{j=1}^{d}\left(a_{i} / z+j\right)^{-1} \\
& =\frac{(-1)^{(d-1)(r+1)} \Theta_{r+1}}{d^{r+1} z^{r+1}} \exp \sum_{k \geqslant 1} \frac{1}{k z^{k}}\left((-1)^{k} \sum_{i} a_{i}^{k} H_{d}^{(k)}-\sum_{i} b_{i}^{k} H_{d-1}^{(k)}\right) .
\end{aligned}
$$

Here $H_{d}^{(k)}:=\sum_{j=1}^{d} j^{-k}$ is the $k$ th harmonic series.

Similarly, in the stable range,

$$
\begin{aligned}
Q(d) I_{d \ell} & =\prod_{i=0}^{r} \prod_{j=\mu_{i}^{\prime}+d_{2}-d+1}^{0}\left(b_{i} / z+j\right) \prod_{j=1}^{\mu_{i}+d}\left(a_{i} / z+j\right)^{-1} \\
& =W_{\beta_{S}, d_{2}}(d) \frac{\Theta_{r+1}}{z^{r+1}} \exp \sum_{k \geqslant 1} \frac{1}{k z^{k}}\left((-1)^{k} \sum_{i} a_{i}^{k} H_{d+\mu_{i}}^{(k)}-\sum_{i} b_{i}^{k} H_{d-d_{2}-\mu_{i}^{\prime}-1}^{(k)}\right)
\end{aligned}
$$

where

$$
W_{\beta_{S}, d_{2}}(d)=(-1)^{\sum_{i=0}^{r}\left(d-\left(d_{2}+\mu_{i}^{\prime}\right)-1\right)} \prod_{i=0}^{r} \frac{\left(d-\left(d_{2}+\mu_{i}^{\prime}\right)-1\right) !}{\left(d+\mu_{i}\right) !}
$$

is the fundamental rational function studied in [Lin10, Wan11]. Here for $r$ even a sign twisting $(-1)^{d}$ is understood.

For a general $d$ (say in the unstable range), the expansion in $1 / z$ depends only on the length data $d+\mu_{i}$ and $d_{2}-d+\mu_{i}^{\prime}$ of the curve class $\beta$. Let $I$ and $J$ be the index set with length less than zero, and let $I^{c}$ and $J^{c}$ be the complementary sets, respectively. Then

$$
\begin{aligned}
Q(d) I_{d \ell}= & \frac{\prod_{i \in I} \prod_{j=\mu_{i}+d+1}^{0}\left(a_{i} / z+j\right)}{\prod_{i \in J} \prod_{j=1}^{\mu_{i}+d}\left(a_{i} / z+j\right)} \frac{\prod_{j=\mu_{i}^{\prime}+d_{2}-d+1}^{0}\left(b_{i} / z+j\right)}{\prod_{i \in J^{c}}^{\mu_{i}^{\prime}+d_{2}-d} \prod_{j=1}^{c}\left(b_{i} / z+j\right)} \\
= & (-1)^{\sum_{i \in I} \mu_{i}+\sum_{i \in J}\left(\mu_{i}^{\prime}+d_{2}\right)+(d-1)(|I|+|J|)} \frac{a_{I} b_{J}}{z^{|I|+|J|}} \\
& \times \frac{\prod_{i \in I}\left(-d-\mu_{i}-1\right) !}{\prod_{i \in I^{c}}\left(d+\mu_{i}\right) !} \frac{\prod_{i \in J}\left(d-d_{2}-\mu_{i}^{\prime}-1\right) !}{\prod_{i \in J^{c}}\left(d_{2}-d+\mu_{i}^{\prime}\right) !} \\
& \times \exp \sum_{k \geqslant 1} \frac{1}{k z^{k}}\left((-1)^{k} \sum_{i \in I^{c}} a_{i}^{k} H_{d+\mu_{i}}^{(k)}+(-1)^{k} \sum_{i \in J^{c}} b_{i}^{k} H_{\mu_{i}^{\prime}+d_{2}-d}^{(k)}\right. \\
& \left.-\sum_{i \in I} a_{i}^{k} H_{-\mu_{i}-d-1}^{(k)}-\sum_{i \in J} b_{i}^{k} H_{d-d_{2}-\mu_{i}^{\prime}-1}^{(k)}\right)
\end{aligned}
$$

This awful-looking expression is in fact very simple in nature. It is a product of $2(r+1)$ series with each one belonging to two types, namely with negative or non-negative length data. 


\section{A.2 Regularization of rational functions}

The key observation is that the whole situation can be considered as a product of $r+1$ series by pairing $\left(L_{i}, L_{i}^{\prime}\right)$ together. As in the Calabi-Yau $P^{1}$ flops case (cf. the proof of Lemma 3.15 in [Wan11]), any factor of the form

$$
\frac{\left(x-\mu^{\prime}-1\right) !}{(x+\mu) !}
$$

(for $x$ a large integer) defines a rational function which has at most simple poles. (Here we take for example $\mu=\mu_{i}$ and $\mu^{\prime}=\mu_{i}^{\prime}+d_{2}$.)

Let $\mu \geqslant-\mu^{\prime}$ (otherwise it is a polynomial and we take Taylor series), then the Laurent series at $x=d \in\left[-\mu, \mu^{\prime}\right] \cap \mathbb{Z}$ is given by

$$
\prod_{j=-\mu}^{\mu^{\prime}}(x-j)^{-1}=\frac{1}{x-d} \prod_{j \neq d ; j=-\mu}^{\mu^{\prime}} \frac{-1}{j-d}\left(\frac{1}{1-(x-d) /(j-d)}\right) .
$$

Taking products over $\left(L_{i}, L_{i}^{\prime}\right)$ shows that the most singular term is actually the product of the simple poles from each $i$. It remains to take into account the harmonic series and figure out the correspondences among them at poles. Replacing $x-d=\Delta x$ by $1 / z$, the above expression splits at $j=d$ and becomes (again using the Taylor series of $\log (1 \pm t)$ )

$$
\frac{1}{z} \frac{(-1)^{\mu^{\prime}-d}}{(\mu+d) !\left(-d+\mu^{\prime}\right) !} \exp \sum_{k \geqslant 1} \frac{1}{k z^{k}}\left((-1)^{k} H_{d+\mu}^{(k)}+H_{\mu^{\prime}-d}^{(k)}\right) .
$$

Notice the formal correspondence with $a_{i}=1$ and $b_{i}=-1$ up to a sign.

The expansion of $W[r+1]$ in $1 / z$ is the Laurent expansion of $W(\vec{x})$ at $\vec{x}=x$. The unstable range contains all possible poles of $W(x)$. The constant term at $x=d$ is the regular part $\operatorname{Reg} W(d)$. In the stable range,

$$
W(d)=\operatorname{Reg} W(d)=(-1)^{(d-1)(r+1)} \frac{\Theta_{r+1}}{d^{r+1}} \prod_{i=0}^{r} \prod_{j=1}^{\mu_{i}}(j+d)^{-1} \prod_{j=1}^{\mu_{i}^{\prime}+d_{2}}(j-d)^{-1},
$$

which by definition coincides with $\Theta_{r+1} W_{\beta_{S}, d_{2}}(d)$.

By the same process, the Taylor expansion at $x=d$ gives back $Q(d) I_{d \ell}$ with $a_{i}=1$ and $b_{i}=-1$. Notice that this does not recover $Q(d) I_{d \ell}$ completely since the process does depend on the presentation of the rational expression. Nevertheless, the above discussions lead to the following.

Lemma A.4. In the full range of $d$, the series expansion

$$
z^{r+1} Q(x) I_{x \ell}=\sum_{k \leqslant r+1} W_{k} z^{k}
$$

and the Laurent expansion of $W_{\beta_{S}, d_{2}}(x)$ in $1 / z$, denoted by $\sum_{k \leqslant r+1} w_{k} z^{k}$, at $x=d$ are compatible in the sense that

$$
w_{k}(d)=\left.W_{k}(d)\right|_{a_{i}=1, b_{i}=-1}
$$

Here is a basic fact concerning the polynomial part of a rational function.

Lemma A.5. Let $F(x)$ be a rational function with poles at $x=e_{j}$ for all $j$ and with polynomial 


\section{INVARIANCE OF QUANTUM RINGS II}

part $P(x)$. Then

$$
P(e)=\operatorname{Reg} F(e)-\sum_{e_{j} \neq e} \operatorname{Pri}_{e_{j}} F(e),
$$

where $\operatorname{Pri}_{e_{j}} F(e)$ is the principal part of $F(x)$ at $x=e_{j}$.

Proof. Let $n_{j}=\operatorname{ord}_{x=e_{j}} F(x)$. By division and taking partial fractions, we have

$$
F(x)=P(x)+\frac{R(x)}{\prod_{j}\left(x-e_{j}\right)^{n_{j}}}=P(x)+\sum_{j} \operatorname{Pri}_{e_{j}} F(x) .
$$

If $e \notin\left\{e_{j}\right\}$, then $\operatorname{Reg} F(e)=F(e)$ and the lemma holds. If $e=e_{i}$ for some $i$, then

$$
F(x)=\operatorname{Pri}_{e} F(x)+\left(P(x)+\sum_{j \neq i} \operatorname{Pri}_{e_{j}} F(x)\right)
$$

and the lemma again holds.

Combining both lemmas leads to results on the first stable series $W_{0}(d)$. For ease of notation, denote by

$$
A=A(q, z)=z^{-\lambda_{\beta}-(r+1)} q^{\beta_{S}} q^{d_{2} \gamma}
$$

the basic factor centered at the first stable series $\left(\lambda_{\beta} \equiv c_{1}(X / S) \cdot \beta\right)$.

Proposition A.6. Given $\left(\beta_{S}, d_{2}\right)$ with $c_{1}(X / S) \cdot \beta \leqslant-(r+1)$, so that the first stable series is located at non-negative $z$-degree, the "partial Birkhoff factorization" up to the first stable series

$$
P_{1}(z) I:=I-A \sum_{r+1 \geqslant k \geqslant 1 ; e} z^{k} q^{e} \widehat{W}_{k}(e) I
$$

leads to polynomial values $P_{\beta_{S}, d_{2}}(d) q^{d}$ at order $z^{-c_{1}(X / S) \cdot \beta-(r+1)}$ in the stable range. This also holds for general $d$ if we consider $\mathscr{F} P_{1}(z) I^{X}-P_{1}^{\prime}(z) I^{X^{\prime}}$. In particular, this leads to analytic continuations of $P_{1}(z) I$ up to $z^{-c_{1}(X / S) \cdot \beta-(r+1)}$.

The compatibility of the partial $B F$ operators $\mathscr{F} P_{1}(z)=P_{1}^{\prime}(z)$ always holds, even for $c_{1}(X / S) \cdot \beta>-(r+1)$. In that case $\mathscr{F} P_{1}(z) I^{X}-P_{1}^{\prime}(z) I^{X^{\prime}}=0$ for all non-negative $z$-degree terms lying over $\left(\beta_{S}, d_{2}\right)$.

Proof. For $1 \leqslant k \leqslant r+1$, a target term with an additional $z^{k}$-power lies in $A W_{k} z^{k} q^{e \ell}$ and takes on the form

$$
A c_{I J}^{k}(e) a_{I} b_{J} z^{k} q^{e \ell}
$$

with $|I|+|J|=r+1-k \leqslant r$. In particular, there is a corresponding $\mathscr{F}$-compatible term on the $X^{\prime}$ side given by $(\mathscr{F} A) c_{I J}^{k}(e) b_{I}^{\prime} a_{J}^{\prime} z^{k} q^{-e \ell^{\prime}}$.

For a divisor $D$, the naive quantization has the effect $\hat{D}=z \partial_{D}=D+z \delta_{D}$, where $\delta_{D}$ is the number operator which acts on $q^{\beta}$ by $\delta_{D} q^{\beta}=(D . \beta) q^{\beta}$. Then in the partial BF procedure (cf. Theorem 2.10)

$$
I-A \sum_{k, e, I, J} c_{I J}^{k}(e) z^{k} q^{e} \prod_{i \in I}\left(a_{i}+z \delta_{a_{i}}\right) \prod_{j \in J}\left(b_{j}+z \delta_{b_{j}}\right) I,
$$

the first term $a_{I} b_{J}$ in the product cancels the target term. 
Modulo higher $\beta_{S}$ and $d_{2} \gamma$, we need to consider only the extremal contribution $\sum_{d \geqslant 1} I_{d \ell} q^{d}$ to the product $\left(q:=q^{\ell}\right)$. The highest $z$ degree comes from

$$
\begin{gathered}
-A c_{I J}^{k}(e) z^{k+(r+1-k)} q^{e} \prod \delta_{a_{i}} \prod \delta_{b_{j}} \sum_{d \geqslant 1} \frac{(-1)^{(d-1)(r+1)} \Theta_{r+1}}{d^{r+1} z^{r+1}} q^{d} \\
=-(-1)^{|J|} A c_{I J}^{k}(e) \Theta_{r+1} \sum_{d \geqslant 1} \frac{(-1)^{(d-1)(r+1)}}{d^{k}} q^{d+e} \\
=-(-1)^{|J|} A c_{I J}^{k}(e) \Theta_{r+1} \sum_{d \geqslant e+1} \frac{(-1)^{(d-e-1)(r+1)}}{(d-e)^{k}} q^{d} .
\end{gathered}
$$

By construction, we have for each fixed $k$ and unstable $e$

$$
\sum_{|I|+|J|=r+1-k}(-1)^{|J|} c_{I J}^{k}(e)=\left.W_{k}(e)\right|_{a_{i}=1, b_{i}=-1}=w_{k}(e) .
$$

If $d$ is in the stable range, then summing all the unstable terms with positive $z$-power gives rise to the principal part of $W_{\beta_{S}, d_{2}}(d)$. Thus the result follows by a careful check of the signs.

If $d$ is in the unstable range, then there are two places in the proof of polynomiality which need to be modified.

First, $W_{0}(d)$ is related to $\operatorname{Reg} W_{\beta_{S}, d_{2}}(d)$ if we set $a_{1}=1$ and $b_{i}=-1$. Alternatively, as $d$ makes sense on both the $X$ and $X^{\prime}$ sides, we also have the relation on the topological defect

$$
\mathscr{F} W_{0}(d)-W_{0}^{\prime}(d)=(-1)^{r+1} \operatorname{Reg} W_{\beta_{S}, d_{2}}(d) \Theta_{r+1}^{\prime},
$$

where $\Theta_{r+1}^{\prime}=\prod_{i=0}^{r} b_{i}^{\prime}=\prod_{i=0}^{r}\left(c_{1}\left(L_{i}\right)+\xi^{\prime}-h^{\prime}\right)$. (This follows from Part I [LLW16]. Indeed, it is clear that the difference is a scalar multiple of $\Theta_{r+1}^{\prime}$ since it is in the kernel of the multiplication map by $\xi^{\prime}$.)

Second, the shifting of a $k$ th order pole by $e$ works only for $e<d$. The poles at $e$ with $e>d$ are missing from the formula on the $X$ side. Thus to receive a complete correction of the principal part from all $e \neq d$ we need to consider $\mathscr{F} P_{1}(z) I^{X / S}-P_{1}^{\prime}(z) I^{X^{\prime} / S}$, and only this.

For the last statement, notice that $\mathbf{f}(q)+\mathbf{f}\left(q^{-1}\right)=(-1)^{r}$ is formally equivalent to the vanishing of the Euler series $E(q):=\sum_{d \in \mathbb{Z}} q^{d}=0$. Hence

$$
\sum_{d \in \mathbb{Z}} P_{\beta_{S}, d_{2}}(d) q^{d}=P_{\beta_{S}, d_{2}}(q d / d q) E(q)=0 .
$$

The proof is complete.

\section{A.3 A remark on higher regularization}

Next we move to the Birkhoff factorization up to the second stable series. This step is needed only if

$$
-c_{1}(X / S) \cdot \beta-(r+1) \geqslant 1
$$

Harmonic series appear naturally and the expected regularization into polynomials becomes much more tricky. A simple useful fact is that the difference of two harmonic series is a rational function.

Let $\lambda_{j}=c_{1}\left(L_{j}\right)$ and $\lambda_{j}^{\prime}=c_{1}\left(L_{j}^{\prime}\right)$. Denote by $e$ an index in the unstable range; then the partial 
$\mathrm{BF}$ with order 1 more reads as

$$
\begin{aligned}
P_{2}(z) I:= & I-A \sum_{r+1 \geqslant k \geqslant 1 ; e} z^{k} q^{e} \widehat{W}_{k}(e) I-A \sum_{d: \text { stable }} q^{d} P_{\beta_{S}, d_{2}}(d) \widehat{\Theta}_{r+1} I \\
& -A \sum_{d: \text { unstable }} q^{d}\left(\widehat{W}_{0}(d)-\sum_{e<d} \operatorname{Pri}_{e}(d) \widehat{\Theta}_{r+1}\right) I,
\end{aligned}
$$

where $\Theta_{r+1}=\prod_{j=0}^{r} b_{j}=\prod_{j=0}^{r}\left(\left(\lambda_{j}+\lambda_{j}^{\prime}\right)+\xi-a_{j}\right)$ and

$$
\widehat{\Theta}_{r+1}:=\prod_{j=0}^{r} z \partial_{b_{j}}-(-1)^{r+1} \prod_{j=0}^{r} z \partial_{a_{j}}
$$

(since $\prod a_{j}=0$, the corresponding quantization product is removed). By construction, the first stable series vanishes automatically.

Now we investigate the second stable series, namely the term with the same degree as

$$
A z^{-1}=z^{-\lambda_{\beta}-(r+1)-1} q^{\beta_{S}} q^{d_{2} \gamma} .
$$

They all contain the factor $(-1)^{(d-1)(r+1)} \Theta_{r+1}$; hence we may remove $\xi$ from the remaining classes.

The main terms come from the first two series in (A.10). The terms from $I$ are degree $A$ terms multiplied by the following harmonic series:

$$
\begin{aligned}
-\sum & a_{i} H_{d+\mu_{i}}-\sum b_{i} H_{d-1-\mu_{i}^{\prime}-d_{2}} \\
= & h \sum\left(-H_{d+\mu_{i}}+H_{d-1-\mu_{i}^{\prime}-d_{2}}\right)-\sum\left(\lambda_{i}+\lambda_{i}^{\prime}\right) H_{d-1} \\
& +\sum \lambda_{i}\left(H_{d}-H_{d+\mu_{i}}\right)+\sum \lambda_{i}^{\prime}\left(H_{d-1}-H_{d-1-\mu_{i}^{\prime}-d_{2}}\right)-\sum \lambda_{i} / d .
\end{aligned}
$$

The terms from the second series form a sum over $k$ and $e$, which has two parts: one with $\left(z \delta_{h}\right)^{r}$ on the second extremal series, which is

$$
\sum(-1)^{|J|} c_{I J}^{k}(e) A z^{-1} \Theta_{r+1}
$$

multiplied by

$$
-\sum a_{i} H_{d}-\sum b_{i} H_{d-1}=-(r+1) h / d-\sum\left(\lambda_{i}+\lambda_{i}^{\prime}\right) H_{d-1}-\sum \lambda_{i} / d,
$$

and another one with one less differentiation $\left(z \delta_{h}\right)^{r-1}$ on the top extremal term, which receives a factor

$$
\left(\sum_{i \in I} a_{i}-\sum_{i \in J} b_{i}\right) / d=(r+1-k) h / d+\sum_{i \in I} \lambda_{i} / d-\sum_{i \in J} \lambda_{i}^{\prime} / d .
$$

For each $(k, e)$, we find a correction factor

$$
-\frac{k h}{d} \quad\left(\mapsto-\frac{k h}{d-e} \quad \text { after a shift by } q^{e}\right) ;
$$

hence this gives rise to a derivative of $(d-e)^{-k}$.

In the stable range, the terms containing the factor $h$ then lead to the derivative, denoted by - here, of the corresponding rational function. Since $f^{\bullet}-\sum g^{\bullet}=\left(f-\sum g\right)^{\bullet}$, they combine to the polynomial

$$
h P_{\beta_{S} \cdot d_{2}}^{\bullet}(d),
$$

which is expected for the purpose of analytic continuations. 


\section{Y.-P. LeE, H.-W. Lin ANd C.-L. WANG}

Similarly, the terms containing the factor $\lambda_{i}+\lambda_{i}^{\prime}$ combine to

$$
-\left(c_{1}+c_{1}^{\prime}\right) P_{\beta_{S}, d_{2}}(d) H_{d-1} .
$$

This is unfortunately the trouble term, due to the appearance of $H_{d-1}$.

Finally, the remaining terms combine to $P_{\beta_{S}, d_{2}}(d)$ multiplied by certain rational functions in $d$. This is a good term since it can be handled by the regularization procedure introduced in Section A.2.

For the unstable range, as in the proof of Proposition A.6, it is expected that a similar calculation holds if we consider $\mathscr{F} P_{2}(z) I^{X / S}-P_{2}^{\prime}(z) I^{X^{\prime} / S}$.

Combining the third series in (A.10) and the one on the $X^{\prime}$ side does produce correction terms, via harmonic convolution, to cancel out the bad term (A.12). The actual calculation is, however, becoming more and more involved.

Simple examples where the higher regularization is carried out explicitly can be found in [LLW12]. But the elementary method used there (harmonic convolution, etc.) does not seem to apply to the general case. This was one of the major motivations for us to develop the quantum Leray-Hirsch theorem during the early stage of this project after [LLW10].

\section{ACKNOWLEDGEMENTS}

We would like to thank the anonymous referee for pointing out several typographical errors in an earlier version of the paper.

\section{REFERENCES}

Bro14 J. Brown, Gromov-Witten invariants of toric fibrations, Int. Math. Res. Not. 2014 (2014), no. 19, 5437-5482; http://dx.doi.org/10.1093/imrn/rnt030.

CG07 T. Coates and A. Givental, Quantum Riemann-Roch, Lefschetz and Serre, Ann. of Math. 165 (2007), no. 1, 15-53; http://dx.doi.org/10.4007/annals.2007.165.15.

Giv04 A.B. Givental, Symplectic geometry of Frobenius structures, in Frobenius manifolds, Aspects Math., vol. E36 (Friedr. Vieweg, Wiesbaden, 2004), 91-112.

Gue08 M. A. Guest, From quantum cohomology to integrable systems, Oxf. Grad. Texts Math., vol. 15 (Oxford Univ. Press, Oxford, 2008).

Lee09 Y.-P. Lee, Notes on axiomatic Gromov-Witten theory and applications, Algebraic Geometry Seattle 2005, Part 1, Proc. Sympos. Pure Math., vol. 80 (Amer. Math. Soc., Providence, RI, 2009), 309-323; http://dx.doi.org/10.1090/pspum/080.1/2483940.

Lin10 H.W. Lin, Quantum invariance under $\mathbb{P}^{1}$ flops of type $(k+2, k)$, Algebraic Geometry in East Asia (Seoul, 2008), Adv. Stud. Pure Math., vol. 6 (Math. Soc. Japan, Tokyo, 2010), 271-300.

LLQW16 Y.-P. Lee, H.-W. Lin, F. Qu and C.-L. Wang, Invariance of quantum rings under ordinary flops III: A quantum splitting principle, Cambridge J. Math. 4 (2016), no. 3, 333-401; http: //dx.doi.org/10.4310/CJM.2016.v4.n3.a2.

LLW10 Y.-P Lee, H.-W. Lin and C.-L. Wang, Flops, motives, and invariance of quantum rings, Ann. of Math. 172 (2010), no. 1, 243-290; http://dx.doi.org/10.4007/annals.2010.172.243.

LLW12_, Analytic continuations of quantum cohomology, Fifth International Congress of Chinese Mathematicians. Part 1, 2, AMS/IP Stud. Adv. Math., vol. 51(1) (Amer. Math. Soc., Providence, RI, 2012), 269-282. 


\section{INVARIANCE OF QUANTUM RINGS II}

LLW16_ Invariance of quantum rings under ordinary flops I: Quantum corrections and reduction to local models, Algebr. Geom. 3, no. 5, (2016), 578-614; http://dx.doi.org/ AG-2016-026.

LP Y.-P. Lee and R. Pandharipande, Frobenius manifolds, Gromov-Witten theory, and Virasoro constraints, available at http://www.math.utah.edu/ yplee/research/.

Wan03 C.-L. Wang, K-equivalence in birational geometry and characterizations of complex elliptic genera, J. Algebraic Geom. 12 (2003), no. 2, 285-306; http://dx.doi.org/10.1090/ S1056-3911-02-00312-0.

Wan04___ K-equivalence in birational geometry, Second International Congress of Chinese Mathematicians, New Stud. Adv. Math., vol. 4 (Int. Press, Somerville, MA, 2004), 199-216.

Wan11 , Quantum invariance under flop transitions, in Geometry and Analysis, no. 2, Adv. Lect. Math., vol. 18 (Int. Press, Somerville, MA, 2011), 347-370.

Yuan-Pin Lee yplee@math.utah.edu

Department of Mathematics, University of Utah, Salt Lake City, Utah 84112-0090, USA

Hui-Wen Lin linhw@math.ntu.edu.tw

Department of Mathematics and Taida Institute of Mathematical Sciences (TIMS), National Taiwan University, Taipei 10617, Taiwan

Chin-Lung Wang dragon@math.ntu.edu.tw

Department of Mathematics, Center for Advanced Studies in Theoretical Sciences (CASTS)

and

Taida Institute of Mathematical Sciences (TIMS), National Taiwan University, Taipei 10617, Taiwan 